\title{
Supporting Information For \\ Urine metabolome profiling reveals imprints of food heating processes after dietary intervention with differently cooked potatoes
}

The supplementary file includes

\section{Supplementary tables}

- Table S1. Characteristics of subjects for the dietary intervention study at the time of enrollment.

- Table S2. Composition of standardized meals and outline of type of standardized meals consumed at the various times.

- Table S3. Preprocessing parameters in MZmine2.

- Table S4. MS/MS fragments of the tentatively identified putative biomarkers for intake of potato chips and French fries and of unknown compounds by LC-Qtof-MS and LTQ-Orbitrap.

$\circ$ Table S5. Coefficient of variation for the intensities of three internal standards after normalization in positive ionization mode.

\section{Supplementary texts}

○ Text S1. The preparation method for test meals.

○ Text S2. LTQ-Orbitrap analysis.

- Text S3. Method for glucuronidase treatment of urine samples.

- Text S4. GC-MS analysis for potato chips.

\section{Supplementary figures}

- Figure S1. Analysis workflow for discovery of intake biomarkers of potato products in UPLC- Qtof-MS.

$\circ \quad$ Figure S2. Summary of PLSDA model performance.

- Figure S3. Overview of selected metabolites for intake of boiled potato, potato chips, French fries and boiled rice.

$\circ \quad$ Figure S4. 2,4-dihydroxy-2,5-dimethyl-3(2H)-furanone with chiral center.

- Figure S5. LC-MS/MS spectra of tentatively identified compounds obtained by LC-Qtof-MS and LTQOrbitrap.

- Figure S6. Extracted ion chromatogram and MS/MS spectrum at 14ev (ESI+) in Qtof of furaneol standard and deconjugated urine sample $2 \mathrm{~h}$ after potato chips intake.

- Figure S7. Chromatogram and mass spectra recorded using the GC-MS after injection of potato chips extract.

- Figure S8. Extracted ion chromatogram and mass spectra recorded using the UPLC-Qtof-MS after injection of pyrraline standard as well as a urine sample collected 1-2 $\mathrm{h}$ after chips intake.

\section{Supplementary references}




\section{Table S1}

Table S1. Characteristics of subjects for the dietary intervention study at the time of enrollment

\begin{tabular}{|l|c|c|}
\hline & $\begin{array}{c}\text { Male } \\
(\mathrm{n}=5)\end{array}$ & $\begin{array}{c}\text { Female } \\
(\mathrm{n}=5)\end{array}$ \\
\hline Age $($ years $)$ & $35.0 \pm 10.75$ & $26.60 \pm 3.07$ \\
\hline Height $(\mathrm{m})$ & $1.82 \pm 0.05$ & $1.67 \pm 0.08$ \\
\hline Weight $(\mathrm{Kg})$ & $83.23 \pm 13.87$ & $60.63 \pm 9.77$ \\
\hline BMI $\left(\mathrm{kg} / \mathrm{m}^{2}\right)$ & $25 \pm 3.51$ & $21.73 \pm 2.07$ \\
\hline Waist $(\mathrm{cm})$ & $92.34 \pm 9.95$ & $74.28 \pm 8.41$ \\
\hline
\end{tabular}

Values are mean $\pm \mathrm{SD}$; BMI, body mass index 


\section{Table S2}

Table S2a. Composition of standardized meals

\begin{tabular}{|c|c|c|c|c|c|c|}
\hline \multicolumn{2}{|l|}{ Breakfast } & \multicolumn{3}{|c|}{ Pasta salad with beans dip } & \multicolumn{2}{|l|}{ Beans stew with pasta } \\
\hline Ciabatta bun, white & 2 & Pasta salad & Pasta, raw (g) & 45 & Pasta, raw (g) & 45 \\
\hline cheese, $45+$ (slices $)$ & 4 & & cauliflower $(\mathrm{g})$ & 30 & white beans ( $\mathrm{g}$ ) & 65 \\
\hline \multirow[t]{2}{*}{ bell pepper, red (full) } & 1 & & cabbage $(\mathrm{g})$ & 80 & cauliflower (g) & 30 \\
\hline & & & carrots $(\mathrm{g})$ & 70 & cabbage $(\mathrm{g})$ & 80 \\
\hline Snacks & & & squash (g) & 70 & carrots $(\mathrm{g})$ & 70 \\
\hline ciabatta bun, refined & ad libitum & Dip & white beans ( $\mathrm{g}$ ) & 25 & squash $(\mathrm{g})$ & 70 \\
\hline \multirow[t]{8}{*}{ cheese, $45+$ (slices) } & ad libitum & & garlic & 1 & garlic & 1 \\
\hline & & & cream cheese $(\mathrm{g})$ & 30 & cream cheese $(\mathrm{g})$ & 30 \\
\hline & & & lemon juice $(\mathrm{g})$ & 2.5 & lemon juice $(\mathrm{g})$ & 4 \\
\hline & & & Basil (g) & 1 & Basil (g) & 1 \\
\hline & & & Salt (g) & 1 & Salt (g) & 1 \\
\hline & & & $\begin{array}{l}\text { Black pepper } \\
\text { (twist) }\end{array}$ & 2 & Black pepper (twist) & 2 \\
\hline & & & & & Water $(\mathrm{g})$ & 50 \\
\hline & & & & & Cheese, $32+$, grated $(\mathrm{g})$ & 20 \\
\hline
\end{tabular}

Table S2b. Outline of type of standardized meals consumed at the various times

\begin{tabular}{l|l|cc}
\hline \multicolumn{2}{l|}{} & Pasta salad & Beans stew with pasta \\
\hline Run in day 2 & Dinner & & ad libitum \\
\hline Test Day & Lunch & $\mathrm{x}$ & $\mathrm{x}$ \\
\hline Follow-up Day 1 & Dinner & $\mathrm{x}$ & $\mathrm{x}$ \\
& Lunch & & \\
\hline
\end{tabular}




\begin{tabular}{|c|c|c|}
\hline Follow-up Day 2 & Breakfast & $\mathrm{x}$ \\
\hline
\end{tabular}
those in ${ }^{1}$ 


\section{Table S3}

Table S3. Preprocessing parameters in MZmine 2

\begin{tabular}{|c|c|c|}
\hline & Batch step & Parameters \\
\hline \multirow{10}{*}{ 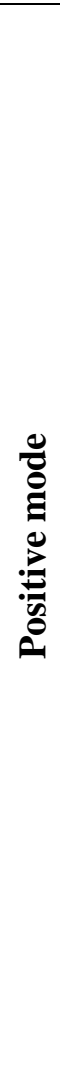 } & Raw data import & \\
\hline & Mass detection & Noise level: 12 \\
\hline & Chromatogram builder & $\begin{array}{l}\text { Min time span }(\mathrm{min}): 0.02 \\
\text { Min height: } 4.0 \mathrm{E} 1 ; \mathrm{m} / \mathrm{z} \text { tolerance: } 0.02 \mathrm{mz} \text { or } 30 \mathrm{ppm}\end{array}$ \\
\hline & Chromatogram deconvolution & $\begin{array}{l}\text { Chromatographic threshold: } 88 \% \text {; Search minimum in RT } \\
\text { range (min): } 0.02 \text {; Minimum relative height: } 1.15 \% \text {; Minimum } \\
\text { absolute height: } 3.8 \mathrm{E} 1 \text {; Min ratio of peak/top edge: } 1.00 \text {; Peak } \\
\text { duration range }(\mathrm{min}): 0.01-0.35\end{array}$ \\
\hline & Isotopic pattern & $\begin{array}{l}\mathrm{m} / \mathrm{z} \text { tolerance: } 0.06 \text { or } 30 \mathrm{ppm} \text {; Retention time tolerance: } 0.01 \text {; } \\
\text { Monotonic shape; maximum charge: } 1\end{array}$ \\
\hline & Duplicate peak filter & $\mathrm{m} / \mathrm{z}$ tolerance: 0.4 or $30 \mathrm{ppm}$; RT tolerance: 0.005 \\
\hline & Duplicate peak filter & $\mathrm{m} / \mathrm{z}$ tolerance: 0.005 or $5 \mathrm{ppm}$; RT tolerance: 0.12 \\
\hline & Join aligner & $\begin{array}{l}\mathrm{m} / \mathrm{z} \text { tolerance: } 0.03 \text { or } 30 \mathrm{ppm} \text {; Absolute retention time } \\
\text { tolerance: } 0.08 ; \text { Weight for both } \mathrm{m} / \mathrm{z} \text { tolerance and retention } \\
\text { time tolerance: } 10\end{array}$ \\
\hline & Peak list rows filter & $\begin{array}{l}\text { Min peaks in a row: } 44 \text { and } 4 \text { for blank } \\
\text { Minimum peaks in an isotope pattern: } 1 ; \mathrm{m} / \mathrm{z} \text { range: } 0-1500 \text {; } \\
\text { RT range: } 0-10 \text {; peak duration range: } 0.01-0.35\end{array}$ \\
\hline & Peak finder & $\begin{array}{l}\text { Intensity tolerance: } 50 \% ; \mathrm{m} / \mathrm{z} \text { tolerance: } 0.03 \text { or } 30 \mathrm{ppm} \text {; } \\
\text { Absolute retention time tolerance: } 0.05\end{array}$ \\
\hline \multirow{7}{*}{ 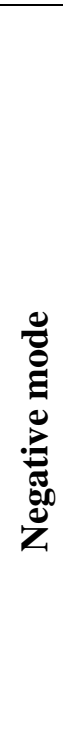 } & Raw data import & \\
\hline & Mass detection & Noise level: 12 \\
\hline & Chromatogram builder & $\begin{array}{l}\text { Min time span }(\mathrm{min}): 0.03 ; \\
\text { Min height: } 4.0 \mathrm{E} 1 ; \mathrm{m} / \mathrm{z} \text { tolerance: } 0.02 \mathrm{mz} \text { or } 30 \mathrm{ppm}\end{array}$ \\
\hline & Chromatogram deconvolution & $\begin{array}{l}\text { Chromatographic threshold: } 90 \% \text {; Search minimum in RT } \\
\text { range (min): } 0.025 \text {; Minimum relative height: } 5 \% \text {; Minimum } \\
\text { absolute height: } 3.5 \mathrm{E} 1 \text {; Min ratio of peak/top edge: } 1.00 \text {; Peak } \\
\text { duration range }(\min ): 0.02-0.35\end{array}$ \\
\hline & Isotopic pattern & $\begin{array}{l}\mathrm{m} / \mathrm{z} \text { tolerance: } 0.06 \text { or } 70 \mathrm{ppm} \text {; Retention time tolerance: } 0.02 \text {; } \\
\text { Monotonic shape; maximum charge: } 1\end{array}$ \\
\hline & Join aligner & $\begin{array}{l}\mathrm{m} / \mathrm{z} \text { tolerance: } 0.02 \text { or } 5 \mathrm{ppm} ; \text { Absolute retention time } \\
\text { tolerance: } 0.2 ; \text { Weight for both } \mathrm{m} / \mathrm{z} \text { tolerance and retention } \\
\text { time tolerance: } 10\end{array}$ \\
\hline & Duplicate peak filter & $\mathrm{m} / \mathrm{z}$ tolerance: 0.002 or $5 \mathrm{ppm}$; RT tolerance: 0.02 \\
\hline
\end{tabular}




\begin{tabular}{|l|l|l|}
\hline & Duplicate peak filter & $\mathrm{m} / \mathrm{z}$ tolerance: 0.08 or $10 \mathrm{ppm}$; RT tolerance: 0.004 \\
\cline { 2 - 3 } & Peak list rows filter & $\begin{array}{l}\text { Min peaks in a row: } 44 \text { and no filter for test samples and } \\
\text { blanks, respectively. } \\
\text { Minimum peaks in an isotope pattern: } 1 ; \mathrm{m} / \mathrm{z} \text { range: } 0-1500 ; \\
\text { RT range: } 0-10 ; \text { peak duration range: } 0.02-0.35\end{array}$ \\
\hline & Peak finder & $\begin{array}{l}\text { Intensity tolerance: } 50 \% ; \mathrm{m} / \mathrm{z} \text { tolerance: } 0.03 \text { or } 30 \mathrm{ppm} ; \\
\text { Absolute retention time tolerance: } 0.05\end{array}$ \\
\hline
\end{tabular}

*m/z: mass to charge ratio; RT: retention time; Intensity: Peak area. 
Table S4

Table S4. MS/MS fragments of the tentatively identified putative biomarkers for intake of potato chips and French fries and of unknown compounds by LC-Qtof-MS and LTQ-Orbitrap

\begin{tabular}{|c|c|c|c|c|c|c|c|c|c|c|}
\hline$\#$ & Suggested metabolite & $\begin{array}{l}\text { MSI } \\
\text { level }\end{array}$ & $\begin{array}{l}\text { Elementary } \\
\text { composition }\end{array}$ & $\begin{array}{l}\text { RT } \\
(\mathbf{m i n})\end{array}$ & $\begin{array}{l}\text { Measured } \\
\text { mass }(\mathrm{m} / \mathbf{z}) \\
{[\mathrm{M}+\mathrm{H}] /[\mathrm{M}-} \\
\mathrm{H}]\end{array}$ & $\begin{array}{l}\text { Fragments by Qtof (mass detector is } \\
\text { VION) }\end{array}$ & Fragments by LTQ-Orbitrap & $\begin{array}{l}\text { PLSDA/A } \\
\text { NOVA-2 }\end{array}$ & $\begin{array}{l}\text { Tentative } \\
\text { ly } \\
\text { identified }\end{array}$ & Source $^{a}$ \\
\hline \multicolumn{11}{|c|}{ Twenty-two metabolites for intake of deep-fried potato products } \\
\hline \multicolumn{11}{|c|}{ Furanone metabolites } \\
\hline M1 & Furaneol glucuronide & 1 & $\mathrm{C}_{12} \mathrm{H}_{16} \mathrm{O}_{9}$ & 4.19 & $305.0888 /-$ & $\begin{array}{l}\text { POS: } \\
\text { 305.0888[M+H]+; } \\
\text { 129.05474[M+H-GLU]+; } \\
\left.\text { 111.0440[M+H-GLU- } \mathrm{H}_{2} \mathrm{O}\right]+; \\
\text { 101.0596[M+H-GLU-CO]+; } \\
\text { 83.04912[M+H-GLU-HCOOH]+; } \\
\left.\text { 57.0330[M+H-GLU-C } \mathrm{H}_{4} \mathrm{O}_{2}\right]+; \\
\text { 55.0541[M+H-GLU-HCOOH-CO]+; }\end{array}$ & 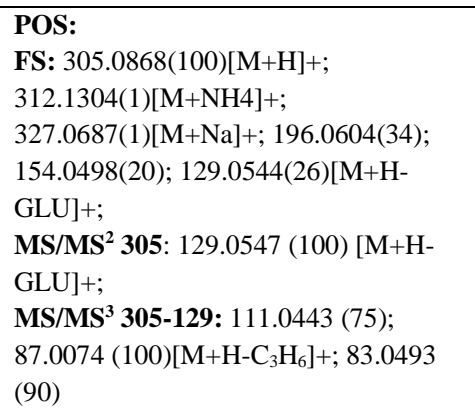 & $x / x$ & $x$ & $\begin{array}{l}\text { Metabolized } \\
\text { component } \\
\text { from potato } \\
\text { chips or } \\
\text { French fries }\end{array}$ \\
\hline M2 & $\begin{array}{l}\text { 2,4-Dihydroxy-2,5- } \\
\text { dimethyl-3(2H)-furanone } \\
\text { glucuronide I }\end{array}$ & 3 & $\mathrm{C}_{12} \mathrm{H}_{16} \mathrm{O}_{10}$ & 2.76 & $\begin{array}{l}145.0506^{\mathrm{b}} / \\
319.0654\end{array}$ & $\begin{array}{l}\text { POS: } \\
\text { 321.0820[M+H]+; } \\
\text { 145.0496[M+H-GLU]+; } \\
\left.\text { 127.0390[M+H-GLU- }{ }_{2} \mathrm{O}\right]+; \\
\text { 99.0439[M+H-GLU-HCOOH]+; } \\
\left.\text { 85.0283[M+H-GLU-COCH }{ }_{2}\right]+; \\
\text { 81.0335[M+H-GLU- } \mathrm{H}_{2}{\left.\mathrm{O}-\mathrm{CO}_{2}\right]+;} \text { 71.0489[M+H-GLU-HCOOH-CO]+; } \\
\text { 55.0177; } \\
\text { NEG: } \\
\text { Non-detected }\end{array}$ & $\begin{array}{l}\text { POS: } \\
\text { FS: } 321.0818(40)[\mathrm{M}+\mathrm{H}]+; \\
\text { 343.0638(10)[M+Na]+; } \\
\text { 359.0378(2)[M+K]+; } \\
\text { 145.0495(18)[M+H-GLU]+; } \\
\text { NEG: } \\
\text { FS: } 319.0717(100)[\mathrm{M}-\mathrm{H}]-; \text { 181.0395(5); } \\
\text { MS/MS 319: 289.0605(100)[M-H- } \\
\text { HCHO]-; 143.0373(5)[M-H-GLU]-; } \\
\left.\text { 113.0264(7)[GLU-H-CH} \mathrm{O}_{3}\right] ; 175.0273 \\
\text { (8)[GLU-H]-; 193.0379 (4); } 231.0544 \\
\text { (5); } 301.0606(8) ;\end{array}$ & $x / x$ & $x$ & $\begin{array}{l}\text { Metabolized } \\
\text { component } \\
\text { from potato } \\
\text { chips or } \\
\text { French fries }\end{array}$ \\
\hline M3 & $\begin{array}{l}\text { 2,4-Dihydroxy-2,5- } \\
\text { dimethyl-3(2H)-furanone } \\
\text { glucuronide II }\end{array}$ & 3 & $\mathrm{C}_{12} \mathrm{H}_{16} \mathrm{O}_{10}$ & 2.48 & $\begin{array}{l}145.0492^{b} / \\
319.0653\end{array}$ & $\begin{array}{l}\text { POS: } \\
\text { 321.0821[M+H]+; } \\
\text { 145.0496[M+H-GLU]+; } \\
\left.\text { 127.0391[M+H-GLU-H } \mathrm{H}_{2} \mathrm{O}\right]+; \\
\text { 99.0439[M+H-GLU-HCOOH]+; } \\
\left.\text { 85.0281[M+H-GLU-COCH }{ }_{2}\right]+; \\
\left.\text { 81.0331[M+H-GLU-H } \mathrm{H}_{2} \mathrm{O}_{-} \mathrm{CO}_{2}\right]+;\end{array}$ & $\begin{array}{l}\text { POS: } \\
\text { FS: } 321.0818(50)[\mathrm{M}+\mathrm{H}]+; \\
343.0637(28)[\mathrm{M}+\mathrm{Na}]+; \\
359.0378(7)[\mathrm{M}+\mathrm{K}]+; \\
\text { 145.0495(10)[M+H-GLU]+; } \\
\text { NEG: }\end{array}$ & $x / x$ & $x$ & $\begin{array}{l}\text { Metabolized } \\
\text { component } \\
\text { from potato } \\
\text { chips or } \\
\text { French fries }\end{array}$ \\
\hline
\end{tabular}




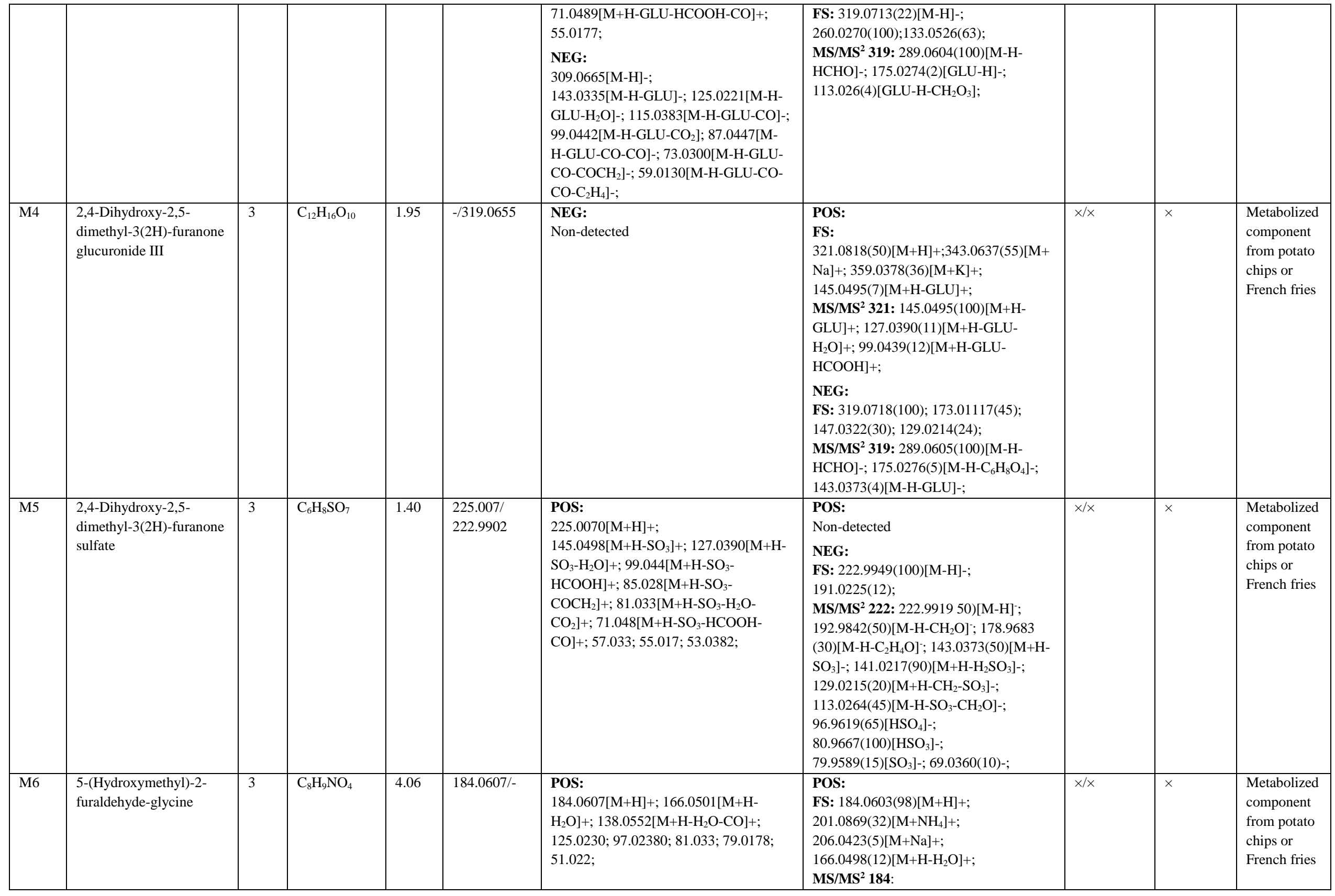




\begin{tabular}{|c|c|c|c|c|c|c|c|c|c|c|}
\hline & & & & & & & $\begin{array}{l}\left.\text { 166.0499(100)[M+H- } \mathrm{H}_{2} \mathrm{O}\right]+; \\
\left.\text { 138.0550(60)[M+H-H }{ }_{2} \mathrm{O}-\mathrm{CO}\right]+; \\
\text { 109.0281(45)[M+H-Glycine]+; } \\
\text { MS/MS } \mathbf{1 8 4 - 1 6 6 - ~ 1 3 8 : ~} 109.0286(100) \\
\text { MS/MS } \mathbf{1 8 4 - 1 6 6 - ~ 1 3 8 - 1 0 9 :} \\
\text { 81.0696(100); 67.0538(80) }\end{array}$ & & & \\
\hline \multicolumn{11}{|c|}{ Pyrrole metabolites } \\
\hline M7 & Pyrraline & 1 & $\mathrm{C}_{12} \mathrm{H}_{18} \mathrm{~N}_{2} \mathrm{O}_{4}$ & 4.18 & 255.1337/- & $\begin{array}{l}\text { POS: } \\
255.1337[\mathrm{M}+\mathrm{H}]+; \\
237.1215\left[\mathrm{M}+\mathrm{H}-\mathrm{H}_{2} \mathrm{O}\right]+; 219.1127[\mathrm{M}+\mathrm{H}- \\
\left.2 \mathrm{H}_{2} \mathrm{O}\right]+; 192.1034\left[\mathrm{M}+\mathrm{H}-\mathrm{H}_{2} \mathrm{O}-\mathrm{CHN}\right]+; \\
175.12334\left[\mathrm{M}+\mathrm{H}-2 \mathrm{H}_{2} \mathrm{O}-\mathrm{CO}_{2}\right]+; \\
158.0968\left[\mathrm{M}+\mathrm{H}-2 \mathrm{H}_{2} \mathrm{O}-\mathrm{CO}_{2}-\mathrm{NH}_{3}\right]+; \\
148.1121\left[\mathrm{M}+\mathrm{H}-2 \mathrm{H}_{2} \mathrm{O}-\mathrm{CO}_{2-}\right. \\
\mathrm{CHN}]+; 143.0729\left[\mathrm{M}+\mathrm{H}_{-}-\mathrm{H}_{2} \mathrm{O}-\mathrm{CO}_{2}-\mathrm{NH}_{3}-\right. \\
\left.\mathrm{CH}_{3}\right]+; 132.08145\left[\mathrm{M}+\mathrm{H}-2 \mathrm{H}_{2} \mathrm{O}-\mathrm{CO}_{2-}\right. \\
\left.\mathrm{NH}_{3}-\mathrm{C}_{2} \mathrm{H}_{2}\right]+; 94.0651 ; 146.0976 ; \\
148.126 ; 118.06 ; 84.0807 ; 67.0540 ;\end{array}$ & 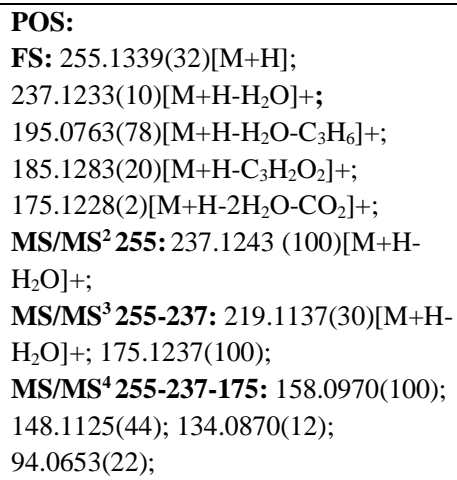 & $x / x$ & $x$ & $\begin{array}{l}\text { Potato chips } \\
\text { or French } \\
\text { fries }\end{array}$ \\
\hline M8 & $\begin{array}{l}\text { N-substituted } \\
\text { formylpyrrole derivative } \\
1\end{array}$ & 3 & $\mathrm{C}_{14} \mathrm{H}_{18} \mathrm{~N}_{2} \mathrm{O}_{4}$ & 5.29 & 279.1479 & $\begin{array}{l}\text { POS: } \\
279.1479[\mathrm{M}+\mathrm{H}]+; 261.1236[\mathrm{M}+\mathrm{H}- \\
\left.\mathrm{H}_{2} \mathrm{O}\right]+; 251.1393[\mathrm{M}+\mathrm{H}-\mathrm{CO}]+; \\
237.1235\left[\mathrm{M}+\mathrm{H}-\mathrm{CO}_{2}\right]+; 219.1128[\mathrm{M}+\mathrm{H}- \\
\left.\mathrm{CO}_{2}-\mathrm{H}_{2} \mathrm{O}\right]+; 191.1185\left[\mathrm{M}+\mathrm{H}-\mathrm{CO}_{2}-\mathrm{H}_{2} \mathrm{O}-\right. \\
\mathrm{CO}]+; 175.1231\left[\mathrm{M}+\mathrm{H}-\mathrm{CO}_{2}-\mathrm{H}_{2} \mathrm{O}-\mathrm{CO}-\right. \\
\left.\mathrm{H}_{2} \mathrm{O}\right]+; 148.1123\left[\mathrm{C}_{10} \mathrm{H}_{13} \mathrm{~N}+\mathrm{H}\right]+; \\
134.0969\left[\mathrm{C}_{9} \mathrm{H}_{2} \mathrm{~N}+\mathrm{H}\right]+; \\
94.0649\left[\mathrm{C}_{6} \mathrm{H}_{7} \mathrm{~N}+\mathrm{H}\right]+; \\
84.0807\left[\mathrm{C}_{5} \mathrm{H}_{9} \mathrm{~N}+\mathrm{H}\right]+; 82.0647\end{array}$ & 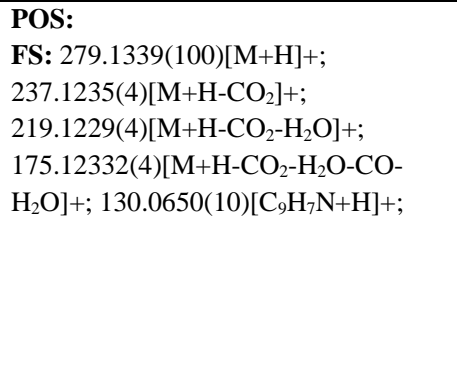 & $x / x$ & $x$ & metabolism \\
\hline M9 & $\begin{array}{l}\text { N-substituted } \\
\text { formylpyrrole derivative } \\
2\end{array}$ & 3 & $\mathrm{C}_{14} \mathrm{H}_{22} \mathrm{~N}_{2} \mathrm{O}_{4}$ & 3.58 & $283.1348 /-$ & 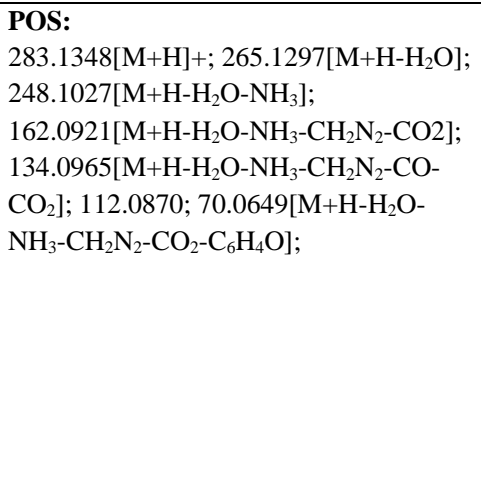 & 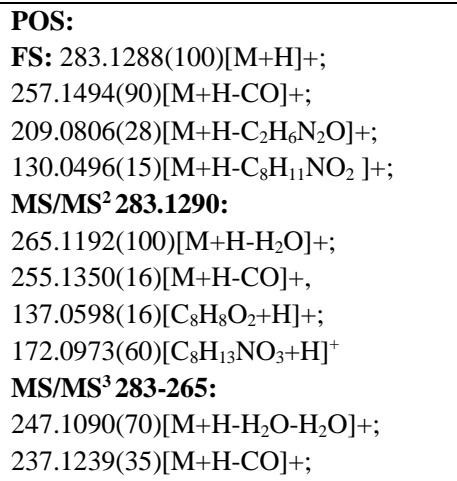 & $x / x$ & $x$ & $\begin{array}{l}\text { Potato chips } \\
\text { or French } \\
\text { fries }\end{array}$ \\
\hline
\end{tabular}




\begin{tabular}{|c|c|c|c|c|c|c|c|c|c|c|}
\hline & & & & & & & $\begin{array}{l}\text { 154.0865(75); 137.0598(100); } \\
129.0662(35)\end{array}$ & & & \\
\hline M10 & $\begin{array}{l}\text { 4-(2-Formyl-5- } \\
\text { (hydroxymethyl)-1H- } \\
\text { pyrrol-1-yl)butanoic acid }\end{array}$ & 3 & $\mathrm{C}_{10} \mathrm{H}_{13} \mathrm{NO}_{4}$ & 5.14 & $\begin{array}{l}212.0917 \\
/ 210.0759\end{array}$ & 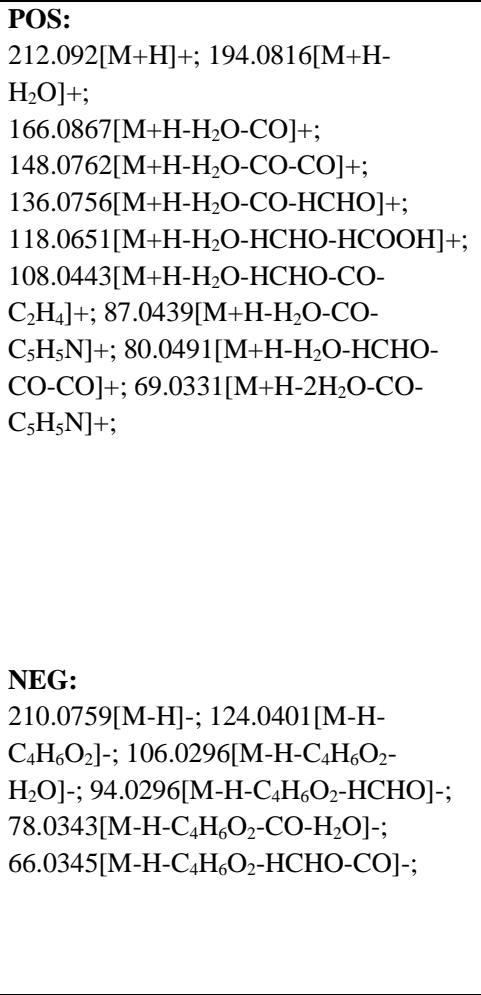 & 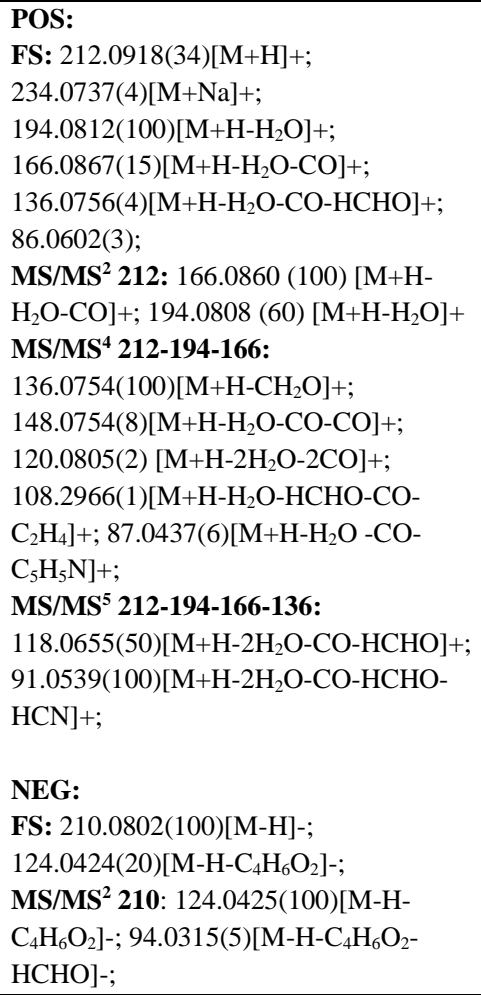 & $x / x$ & $x$ & $\begin{array}{l}\text { Potato chips } \\
\text { or French } \\
\text { fries }\end{array}$ \\
\hline M11 & $\begin{array}{l}\text { 5-(Hydroxymethyl)-1H- } \\
\text { pyrrole-2-carbaldehyde } \\
\text { glucuronide }\end{array}$ & 3 & $\mathrm{C}_{12} \mathrm{H}_{15} \mathrm{NO}_{8}$ & 4.20 & $-/ 300.0690$ & $\begin{array}{l}\text { NEG: } \\
\text { 300.0690[M-H]-; 124.0390[M-H-GLU]-; } \\
\text { 94.0275[M-H-GLU-HCHO]-; 82.029[M- } \\
\text { H-GLU-COCH }]-; \text { 66.0331[M-H-GLU- } \\
\text { HCHO-CO]-; 54.034[M-H-GLU- } \\
\text { COCH }_{2} \text {-HCHO]-; }\end{array}$ & $\begin{array}{l}\text { NEG: } \\
\text { FS:300.0766(100)[M-H] } ; \\
253.1230(40)-; 124.0424(11)[\mathrm{M}-\mathrm{H}- \\
\text { GLU]-; }\end{array}$ & $x / x$ & $x$ & Metabolism \\
\hline
\end{tabular}




\begin{tabular}{|c|c|c|c|c|c|c|c|c|c|c|}
\hline & & & & & & & $\begin{array}{l}\text { MS/MS }{ }^{2} \text { 300:175.0275(76)[GLU-H]-; } \\
\text { 124.0424(32)[M-H-GLU]-; } \\
\left.\text { 113.0263(100)[M-H-C }{ }_{6} \mathrm{H}_{7} \mathrm{NO}_{2}-\mathrm{CH}_{2} \mathrm{O}_{3}\right]-; \\
\text { MS/MS }{ }^{3} \text { 300-124: } 94.0312(100)[\mathrm{M}-\mathrm{H}- \\
\text { GLU-HCHO]-; 82.0314(46) [M-H-GLU- } \\
\left.\mathrm{COCH}_{2}\right]-;\end{array}$ & & & \\
\hline \multicolumn{11}{|c|}{ Pyrazine metabolites } \\
\hline M12 & $\begin{array}{l}\text { 4-(6-methyl-2-pyrazinyl)- } \\
\text { 1,2,3-Butanetriol }{ }^{\mathrm{c}}\end{array}$ & 2 & $\mathrm{C}_{9} \mathrm{H}_{14} \mathrm{~N}_{2} \mathrm{O}_{3}$ & 3.82 & 199.1069/- & 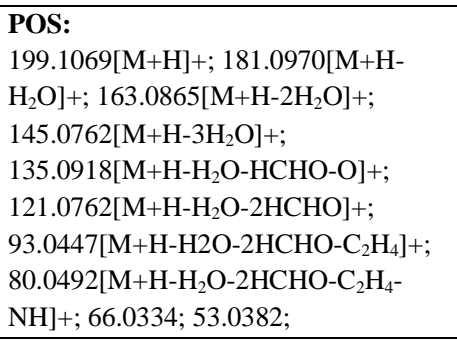 & 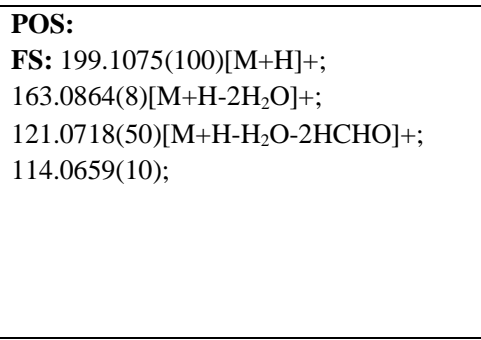 & $x / x$ & $x$ & $\begin{array}{l}\text { Potato chips } \\
\text { or French } \\
\text { fries }\end{array}$ \\
\hline M13 & Pyrazine derivative 1 & 3 & $\mathrm{C}_{8} \mathrm{H}_{12} \mathrm{~N}_{2} \mathrm{O}_{3}$ & 2.18 & 185.0926 & $\begin{array}{l}\text { POS: } \\
\text { 185.0926[M+H]+; 167.0820[M+H- } \\
\text { H2O]+; 149.0710[M+H-H2O-H2O]+; } \\
\text { 137.0711[M+H-CH4O]+; } \\
\text { 121.0761[M+H-2H2O-CO]+; } \\
\text { 108.0679[C3H9NO3+H]+; 93.0449; }\end{array}$ & Non-detected & $x / x$ & $x$ & $\begin{array}{l}\text { Potato chips } \\
\text { or French } \\
\text { fries }\end{array}$ \\
\hline M14 & Pyrazine derivative 2 & 3 & $\mathrm{C}_{8} \mathrm{H}_{10} \mathrm{~N}_{2} \mathrm{O}_{3}$ & 3.81 & $183.0753 /-$ & 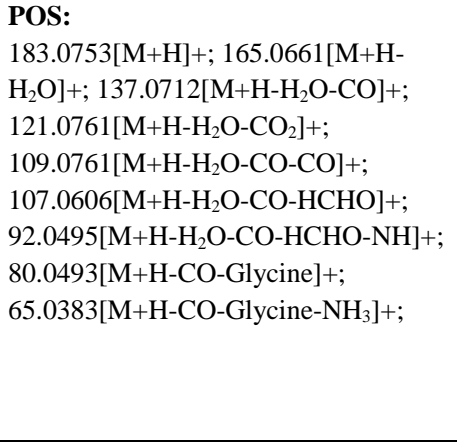 & 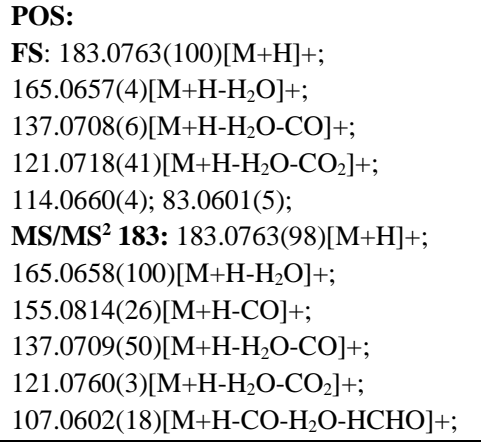 & $1 \times$ & $x$ & $\begin{array}{l}\text { Metabolized } \\
\text { component } \\
\text { from potato } \\
\text { chips or } \\
\text { French fries }\end{array}$ \\
\hline \multicolumn{11}{|c|}{ Unknown metabolites } \\
\hline M15 & Unknown & 4 & $\mathrm{C}_{9} \mathrm{H}_{11} \mathrm{NO}_{2}$ & 4.83 & $180.0679 /-$ & $\begin{array}{l}\text { POS: } \\
\text { 180.0679[M+H]+; 105.03370[M+H- } \\
\text { Glycine]+; 77.03860[M+H-Glycine- } \\
\text { CO]+; }\end{array}$ & $\begin{array}{l}\text { POS: } \\
\text { FS: } 180.0653(100)[\mathrm{M}+\mathrm{H}]+; \\
\text { 2020474(1)[M+NH} 4]+; \\
\text { 105.0333(21)[M+H-Glycine]+; } \\
\text { MS/MS } \mathbf{2} \text { 180: 162.0547(24)[M+H- } \\
\left.\mathrm{H}_{2} \mathrm{O}\right]+; \text { 105.0332(100)[M+H-Glycine]+; } \\
\text { MS/MS }{ }^{3} \text { 180-105: } 95.0489(55) ; \\
\text { 77.0383(100)[M+H-Glycine-CO]+; }\end{array}$ & $/ x$ & & Metabolism \\
\hline
\end{tabular}




\begin{tabular}{|c|c|c|c|c|c|c|c|c|c|}
\hline M16 & $\begin{array}{l}\text { 5-(Hydroxymethyl)-1H- } \\
\text { pyrrole-2-carbaldehyde } \\
\text { derivative glucuronide }\end{array}$ & 3 & $\mathrm{C}_{21} \mathrm{H}_{23} \mathrm{NO}_{11}$ & 4.59 & $-/ 464.1187$ & $\begin{array}{l}\text { NEG: } \\
\text { 464.1187[M-H]-; 288.0873[M-H-GLU]-; } \\
\left.\text { 244.0993[M-H-GLU-CO }{ }_{2}\right]-; \\
\left.\text { 270.0926[M-H-GLU- } \mathrm{H}_{2} \mathrm{O}\right]-; \\
\left.\text { 228.0923[M-H-GLU- } \mathrm{H}_{2} \mathrm{O}-\mathrm{COCH} \mathrm{CH}_{2}\right]-; \\
\left.\text { 226.0825[M-H-GLU-CO }{ }_{2}-\mathrm{H}_{2} \mathrm{O}\right]-; \\
\text { 124.0402[M-H-GLU-CO }{ }_{2}-\mathrm{H}_{2} \mathrm{O}- \\
\left.\mathrm{C}_{3} \mathrm{H}_{6} \mathrm{~N}_{2} \mathrm{O}_{2}\right]-; 106.0296\left[\mathrm{M}-\mathrm{H}-\mathrm{GLU}-\mathrm{CO}_{2}-\right. \\
\left.\mathrm{H}_{2} \mathrm{O}-\mathrm{C}_{3} \mathrm{H}_{6} \mathrm{~N}_{2} \mathrm{O}_{2}-\mathrm{H}_{2} \mathrm{O}\right]-; 94.0294[\mathrm{M}-\mathrm{H}- \\
\left.\mathrm{GLU}_{-} \mathrm{CO}_{2}-\mathrm{H}_{2} \mathrm{O}-\mathrm{C}_{3} \mathrm{H}_{6} \mathrm{~N}_{2} \mathrm{O}_{2}-\mathrm{HCHO}\right]-; \\
\text { 78.0343[M-H-GLU-CO }{ }_{2}-\mathrm{H}_{2} \mathrm{O}- \\
\left.\mathrm{C}_{3} \mathrm{H}_{6} \mathrm{~N}_{2} \mathrm{O}_{2}-\mathrm{CO}-\mathrm{H}_{2} \mathrm{O}\right]-; 66.0343[\mathrm{M}-\mathrm{H}- \\
\text { GLU-CO } \\
\text { CO]-; }\end{array}$ & 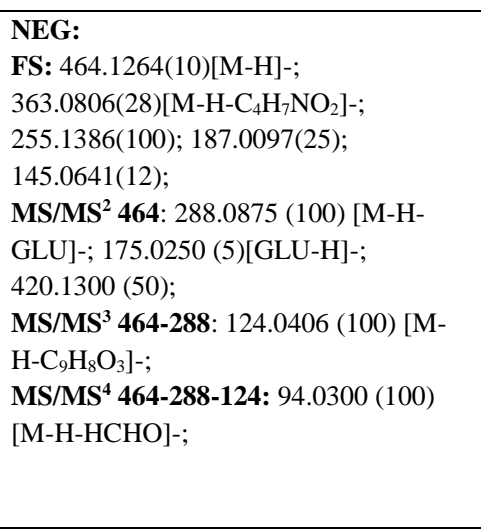 & $/ x$ & Metabolism \\
\hline M17 & $\begin{array}{l}\text { Unknown } \\
\text { Possible proposal: 4-(2- } \\
\text { Formyl-5- } \\
\text { (hydroxymethyl)-1H- } \\
\text { pyrrol-1-yl)butanoic acid }\end{array}$ & 3 & $\mathrm{C}_{16} \mathrm{H}_{21} \mathrm{NO}_{10}$ & 4.75 & $-/ 386.1274$ & $\begin{array}{l}\text { NEG: } \\
\text { Non-detected }\end{array}$ & 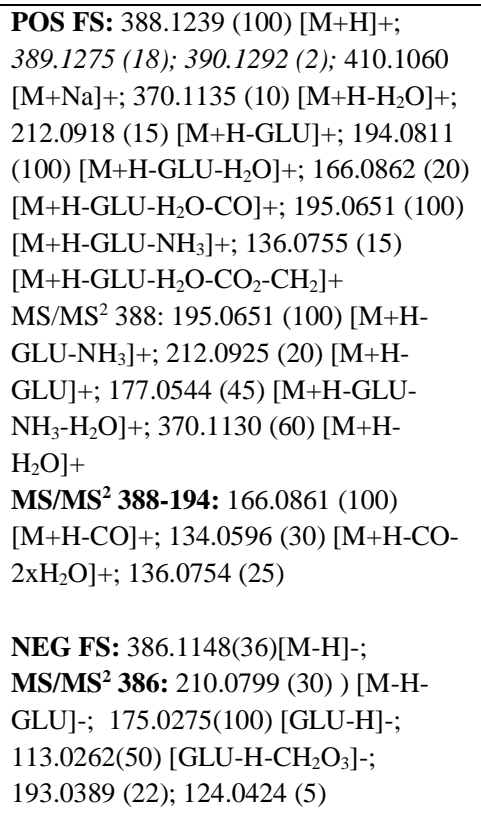 & $/ x$ & Metabolism \\
\hline M18 & Unknown & 4 & & 4.92 & $456.215 /-$ & $\begin{array}{l}\text { POS: } \\
\text { Non-detected }\end{array}$ & $\begin{array}{l}\text { POS: } \\
\text { Non-detected }\end{array}$ & $/ x$ & Metabolism \\
\hline
\end{tabular}




\begin{tabular}{|c|c|c|c|c|c|c|c|c|c|c|}
\hline & & & & & & & $\begin{array}{l}\text { (30)[M-H-CO]-; } 159.0820 \text { (45) [M-H- } \\
\left.\mathrm{H}_{2} \mathrm{O}\right]-;\end{array}$ & & & \\
\hline M20 & $\begin{array}{l}\text { Unknown } \\
\text { Possible proposals: } \\
>\text { 5-Hexyltetrahydro-2- } \\
\text { oxo-3-furan } \\
\text { carboxylic acid } \\
>\text { 5-(1,3-Dimethylbutyl) } \\
\text { tetrahydro-2-oxo-3- } \\
\text { furancarboxylic acid } \\
>\text { 6-Pentyltetrahydro-2- } \\
\text { oxo-2H-pyran-3- } \\
\text { carboxylic acid }\end{array}$ & 4 & $\mathrm{C}_{11} \mathrm{H}_{18} \mathrm{O}_{4}$ & 6.33 & $-/ 213.1132$ & NEG: & 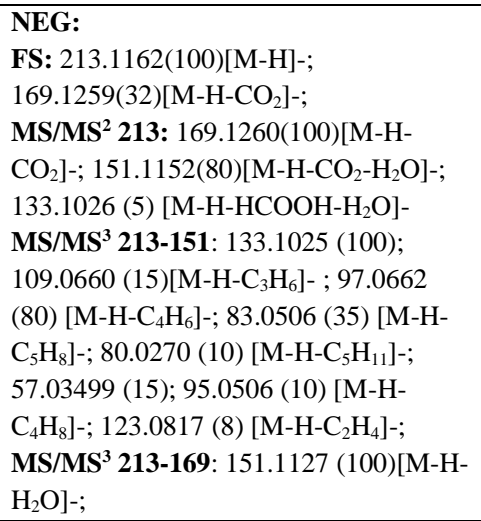 & $/ x$ & & Metabolism \\
\hline M21 & $\begin{array}{l}\text { Unknown glucuronide } \\
\text { Possible proposals: } \\
>\text { 5-Hexyltetrahydro-2- } \\
\text { oxo-3-furan } \\
\text { carboxylic acid } \\
>\text { 5-(1,3-Dimethylbutyl) } \\
\text { tetrahydro-2-oxo-3- } \\
\text { furancarboxylic acid } \\
\text { 6-Pentyltetrahydro-2- } \\
\text { oxo-2H-pyran-3- } \\
\text { carboxylic acid) }\end{array}$ & 4 & $\mathrm{C}_{17} \mathrm{H}_{26} \mathrm{O}_{10}$ & 5.61 & $-/ 389.1457$ & NEG: & $\begin{array}{l}\text { NEG: } \\
\text { FS: 389.1507(15)[M-H]-; } \\
\text { 187.0096(100); 173.0844(70) } \\
\text { MS/MS } \mathbf{3} \text { 389: 213.1137(100)[M-H- } \\
\text { GLU]-; 175.0273(60)[GLU-H]-; } \\
\left.\text { 113.0263(50)[M-GLU-H-CH } \mathrm{O}_{3}\right]-; \\
\text { MS/MS } 3 \text { 389-213: 169.1234(100)[M-H- } \\
\left.\text { GLU-CO } \mathrm{CO}_{2}\right]-; 151.1131(80)[\mathrm{M}-\mathrm{GLU}-\mathrm{H}- \\
\left.\mathrm{CO}_{2}-\mathrm{H}_{2} \mathrm{O}\right]-;\end{array}$ & $/ x$ & & Metabolism \\
\hline M22 & Unknown & 4 & $\mathrm{C}_{10} \mathrm{H}_{14} \mathrm{O}_{5}$ & 5.56 & $-/ 213.077$ & NEG: & $\begin{array}{l}\text { NEG: } \\
\text { FS: } 213.0798(50)[\mathrm{M}-\mathrm{H}]-; \\
178.0536(100)\end{array}$ & $/ x$ & & Metabolism \\
\hline \multicolumn{11}{|c|}{ Two metabolites differentiated intake of potato chips and French fries } \\
\hline M5 & $\begin{array}{l}\text { 2,4-Dihydroxy-2,5- } \\
\text { dimethyl-3(2H)-furanone } \\
\text { sulfate }\end{array}$ & 3 & $\mathrm{C}_{6} \mathrm{H}_{8} \mathrm{SO}_{7}$ & 1.40 & $225.0070^{\mathrm{d} /-}$ & $\begin{array}{l}\text { POS: } \\
225.0070[\mathrm{M}+\mathrm{H}]+; \\
145.0498\left[\mathrm{M}+\mathrm{H}-\mathrm{SO}_{3}\right]+; 127.0390[\mathrm{M}+\mathrm{H}- \\
\left.\mathrm{SO}_{3}-\mathrm{H}_{2} \mathrm{O}\right]+; 99.044\left[\mathrm{M}+\mathrm{H}_{-}-\mathrm{SO}_{3}-\right. \\
\mathrm{HCOOH}]+; 85.028\left[\mathrm{M}+\mathrm{H}_{-}-\mathrm{SO}_{3}-\right. \\
\left.\mathrm{COCH}_{2}\right]+; 81.033\left[\mathrm{M}+\mathrm{H}_{-}-\mathrm{SO}_{3}-\mathrm{H}_{2} \mathrm{O}-\right. \\
\left.\mathrm{CO}_{2}\right]+; 71.048\left[\mathrm{M}+\mathrm{H}-\mathrm{SO}_{3}-\mathrm{HCOOH}-\right. \\
\mathrm{CO}]+; 57.033 ; 55.017 ; 53.0382 ;\end{array}$ & 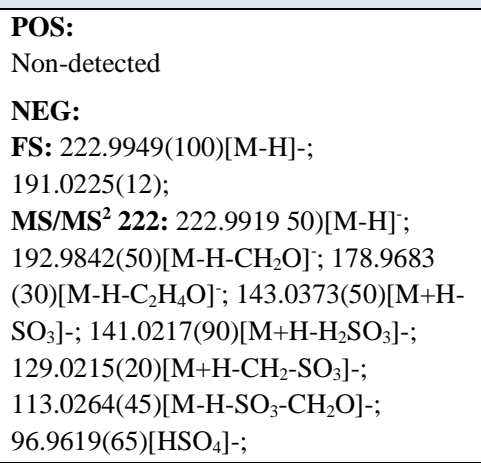 & $x / x$ & $x$ & $\begin{array}{l}\text { Metabolized } \\
\text { component } \\
\text { from potato } \\
\text { chips or } \\
\text { French fries }\end{array}$ \\
\hline
\end{tabular}




\begin{tabular}{|c|c|c|c|c|c|c|c|c|}
\hline & & & & & & $\begin{array}{l}80.9667(100)\left[\mathrm{HSO}_{3}\right]-; \\
79.9589(15)\left[\mathrm{SO}_{3}\right]-; 69.0360(10)-;\end{array}$ & & \\
\hline M23 & Unknown & 4 & 0.69 & $203.0534 /-$ & $\begin{array}{l}\text { NEG: } \\
\text { Non-detected }\end{array}$ & & $/ \times$ & Metabolism \\
\hline \multicolumn{9}{|c|}{ Three metabolites differentiated intake of potato in general and boiled rice } \\
\hline M24 & Unknown & 4 & 1.48 & $116.0703 /$ & $\begin{array}{l}\text { POS: } \\
\text { Non-detected }\end{array}$ & & $1 \times$ & Metabolism \\
\hline M25 & Unknown & 4 & 6.30 & $-/ 331.1415$ & $\begin{array}{l}\text { NEG: } \\
\text { Non-detected }\end{array}$ & & $/ x$ & Metabolism \\
\hline M26 & Unknown & 4 & 6.54 & $-/ 491.2312$ & $\begin{array}{l}\text { NEG: } \\
\text { Non-detected }\end{array}$ & & /x & Metabolism \\
\hline
\end{tabular}

${ }^{a}$ Source of unconjugated compound; ${ }^{b}$ the parent ion 321.08 was found in the raw data but was not present in data after preprocessing so only the product ions were selected during the data analysis; structural identification agrees with 'Wakamatsu et. al. $2019^{2}$ (MS/MS information in supplemental information Figure S5); ${ }^{\mathrm{d}}$ the biomarker could also distinguish the group of deep-fried potato products from boiled potatoes. Abbreviation: GLU, glucuronide; MSI, Metabolomics Standard Initiative; FS: full scan; POS, positive ionization mode; NEG, negative ionization mode. 


\section{Table S5}

Table S5. Coefficient of variation for the intensities of three internal standards after normalization in positive ionization mode

\begin{tabular}{|c|c|c|c|}
\hline \multirow{2}{*}{$\begin{array}{c}\text { Normalization } \\
\text { methods }\end{array}$} & ${ }^{13} \mathrm{C}_{6}$-hippuric acid & Para-aminobenzoic acid & L-tryptophan-d5(indole-d5) \\
\cline { 2 - 4 } & 36.26 & 22.94 & 21.62 \\
\hline $\begin{array}{c}\text { Before } \\
\text { normalization }\end{array}$ & 22.80 & 19.48 & 11.88 \\
\hline Unit-length & 21.00 & 17.00 & 11.50 \\
\hline $\begin{array}{c}\text { Unit-length+batch- } \\
\text { correction }\end{array}$ & & & \\
\hline
\end{tabular}

The three internal standards are ${ }^{13}$ C6-hippuric acid, para-aminobenzoic acid and L-tryptophan-d5(indole-d5). The coefficient of variance (CV\%) for three standards before normalization, after unit-length normalization, and after unit-length and batch-correction is shown in Table S5. The results indicate that the variability for the dataset normalized by unit-length was decreased, and the variation between batches has been slightly corrected by later batch-correction. 


\section{Text S1 Preparation method for test meals}

Potato and meat were combined, each in random order in such a way that all possible combinations of protein and starchy foods were covered in a balanced way across subjects to avoid the foods to influence each other. For the starchy foods, the meals contained BP, PC, FF, or boiled, polished rice. BP was peeled and boiled in water; for PC and FF, they were also peeled and cut in rods or flakes and fried according to procedures similar to commercial products. Fresh Safari variety of potatoes bought from the Lammefjord area, Zealand, Denmark, was used for all processed products and rapeseed oil was used for frying and added to the meals with BP and white rice to assure that all four meals were iso-caloric and contained the same type and amount of fat. 


\section{Text S2 LTQ-Orbitrap analysis}

Urine samples from dietary intervention study were analysed as follows: LC analysis was performed on a Dionex Ultimate 3000 HPLC system coupled with a hybrid linear ion trap Fourier Transform (LTQ FT) Orbitrap high-resolution mass spectrometer (Thermo Fisher Scientific, Waltham, MA, USA) by an electrospray ionization (ESI) probe for MS and $\mathrm{MS}^{\mathrm{n}}$ analysis both in positive and negative ionization modes.

The chromatographic analysis was performed on a Kinetex C18 column (150 mm x $2.1 \mathrm{~mm}$ I.D., particle size $2.6 \mu \mathrm{m}$ ) and a guard column containing the same stationary phase (Phenomenex, Torrance, CA, USA). Column temperature was set at $40^{\circ} \mathrm{C}$. Water (eluent A) and acetonitrile (eluent B), both with $0.1 \%$ formic acid, were used as mobile phases. For chromatographic analysis the following gradient was adopted: 0-1 min isocratic 5\% B, 1-7 min linear gradient 5-45\% B, 7-8.5 min linear gradient 45-80\% B, 8.5-10.5 min isocratic 80\% B, 10.5 -11 min linear gradient $80-5 \% \mathrm{~B}, 11-12 \mathrm{~min} 5 \% \mathrm{~B}$. The flow rate was $350 \mu \mathrm{L} / \mathrm{min}$ and the injection volume was 5 $\mu \mathrm{L}$.

The ESI conditions in positive (and negative) mode were: spray voltage $5.0 \mathrm{kV}(-3.5 \mathrm{kV})$, heated capillary temperature $320^{\circ} \mathrm{C}$, capillary voltage $35 \mathrm{~V}(-35 \mathrm{~V})$ and tube lens $110 \mathrm{~V}(-110 \mathrm{~V})$. In the LTQ component of the instrument, nitrogen was used as both the sheath gas (35 U) and auxiliary gas (5 U), and helium was used as the damping gas. All measurements were done using the automatic gain control of the LTQ to adjust the number of ions entering the trap. Mass calibration was performed with every sequence run just prior to starting the batch by using flow injection of the manufacturer's calibration standards mixture allowing for mass accuracies lower than 5 ppm in external calibration mode.

Each urine sample was analysed, both under positive and negative ionization, using two different mass acquisition methods for each ionization mode. The first method consisted of a full scan (mass range from 100 to $1000 \mathrm{Da}$ ) at a mass resolution of 30,000 FWHM (full width at half maximum for $\mathrm{m} / \mathrm{z} 400$ ) in centroid mode. Then, based on the data dependent acquisition (DDA) mode, the LC-MS/MS analysis of each sample was performed in order to achieve the mass fragmentation spectra. In this method during the chromatographic run, both full scan and $\mathrm{MS}^{2}$ spectra of the 3 most intense ions of each full scan were acquired. The resolving power for $\mathrm{MS}^{2}$ scans was 7500 . Product ions were generated in the LTQ trap at collision energy $35 \mathrm{eV}$ using an isolation width of $2 \mathrm{Da}$. Such double injections with two different mass spectrometry acquisition methods allowed for the collection of high-quality full scan data, while second injection with DDA modality allowed for an automatic collection of high number of $\mathrm{MS}^{2}$ and pseudo $\mathrm{MS}^{\mathrm{n}}$ spectra. In addition, whenever necessary $\mathrm{MS}^{3}$ and $\mathrm{MS}^{\mathrm{n}}$ experiments were carried out for selected ions. 


\section{Text S3 Method for glucuronidase treatment of urine samples}

Glucuronidase treatment of urine samples was performed according to the method described previously ${ }^{3}$. Each urine sample $(20 \mu \mathrm{l})$ was diluted in $80 \mu \mathrm{l} 0.1 \mathrm{M}$ sodium phosphate buffer $(\mathrm{pH}=7.4)$ and incubated with $\beta$ -

glucuronidase from E.coli $\mathrm{K} 12$ (Roche Diagnostics $\mathrm{GmbH}$ ) at $37^{\circ} \mathrm{C}$ for 2 hours, then diluted with $400 \mu 10.01 \%$ and inject onto the UPLC-MS for analysis and compared to the authentic standard. 


\section{Text S4 GC-MS analysis for potato chips}

Volatile compounds were detected and analyzed by using a Thermo Scientific ${ }^{\mathrm{TM}}$ TSQ Quantum ${ }^{\mathrm{TM}}$ GC Triple Quadropole GC-MS. $1 \mathrm{~mL}$ of sample was prepared in a headspace $20 \mathrm{~mL}$ vial. The samples were incubated for 5 $\min$ at $40^{\circ} \mathrm{C}$. The volatile compounds were collected on a Divinylbenzene/Carboxen/Polydimethylsiloxane $2 \mathrm{~cm}$ fibre (DVB-CAR-PDMS Supelco) for an extraction time of $30 \mathrm{~min}$. A VF-wax column (Agilent) $30 \mathrm{~m} / \mathrm{I} . \mathrm{D} 0.25$ $\mathrm{mm} /$ Film $0.25 \mu \mathrm{m}$, was used for the separation. The oven was kept at $40{ }^{\circ} \mathrm{C}$ for 4 min then increased by 6 ${ }^{\circ} \mathrm{C} / \mathrm{min}$ to $250{ }^{\circ} \mathrm{C}$ and kept at the final temperature for $5 \mathrm{~min}$. The injector and interface temperatures were kept at $250{ }^{\circ} \mathrm{C}$ as well. Helium was used as the carrier gas with a flow rate of $1.2 \mathrm{ml} / \mathrm{min}$. The time for thermal desorption of analytes was $4 \mathrm{~min}$. The MS detector was operated in full scan mode at $70 \mathrm{eV}$ with a scan range from 40 to $350 \mathrm{~m} / \mathrm{z}$. Data analysis was performed using Thermo Scientific ${ }^{\mathrm{TM}}$ Xcalibur $^{\mathrm{TM}}$ (Version 2.2 SP1.48, Thermo scientific). Identification of compounds was based on comparison with a mass spectral database (NIST 2.0, Wiley 8). 


\section{Figure S1}

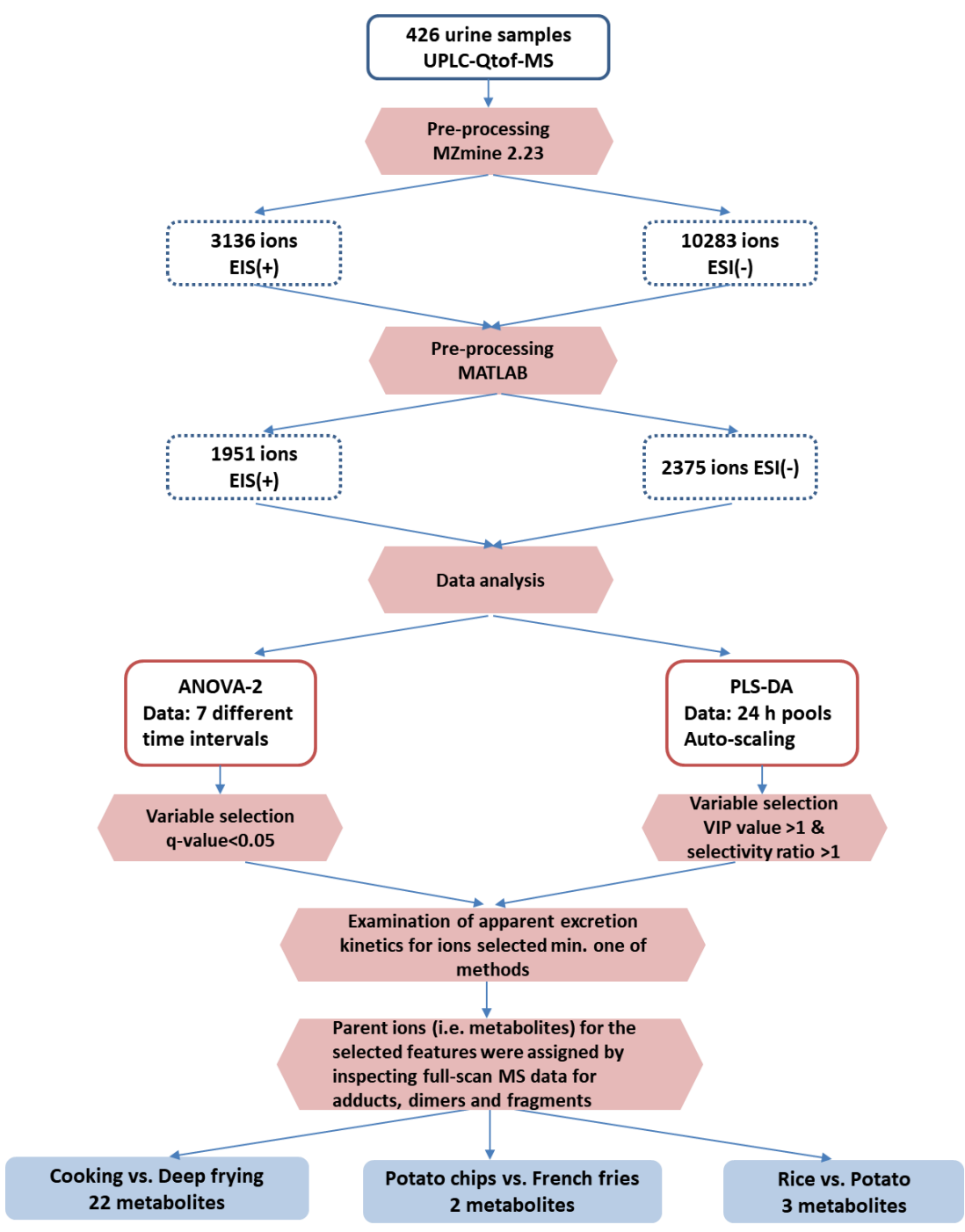

Figure S1. Analysis workflow for discovery of intake biomarkers of potato products in UPLC- Qtof-MS. 


\section{Figure S2}

a. Parameters of misclassification error (ER) and area under the curve (AUC) after validation in positive and negative ionization modes for potato chips vs boiled potato modeling are listed below. The mean values of ER, AUC, $\mathrm{R}^{2}$ and $\mathrm{Q}^{2}$ in 45 models in positive and negative modes were $0.11,0.94,0.94$ and $0.68,0.07,1,0.94$ and 0.88 , respectively.

Positive mode

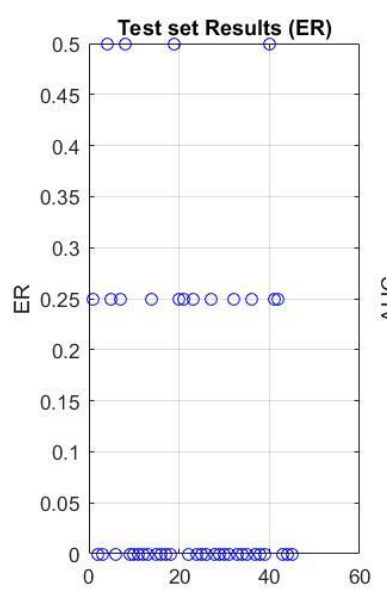

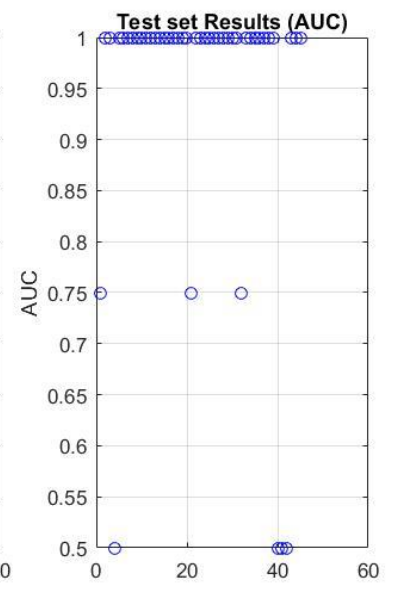

Negative mode

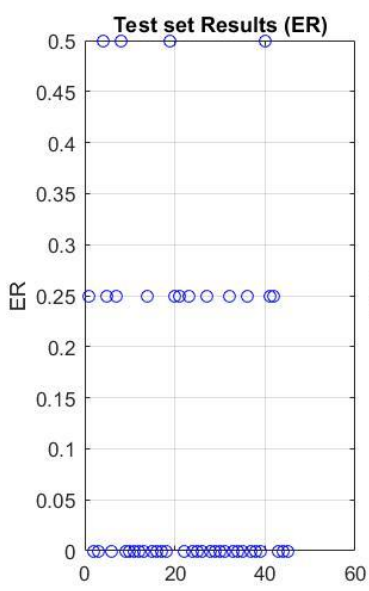

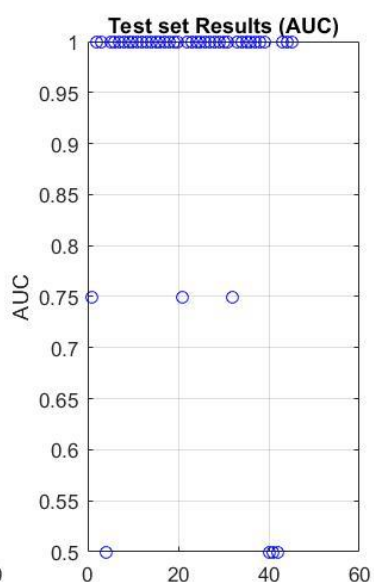

b. Parameters of misclassification error (ER) and area under the curve (AUC) after validation in positive and negative ionization mode for French fries vs boiled potato modeling is listed below. The mean values of ER, AUC, $\mathrm{R}^{2}$ and $\mathrm{Q}^{2}$ in 45 models in positive and negative mode were $0.21,0.92,0.89$ and $0.63,0.51,0.54,0.87$ and 0.22 , respectively. The parameters indicate that the model for negative mode is not appropriate for classification, and ANOVA-2 was therefore the main approach for variable selection to differentiate the groups of boiled potato and French fries.

Positive mode

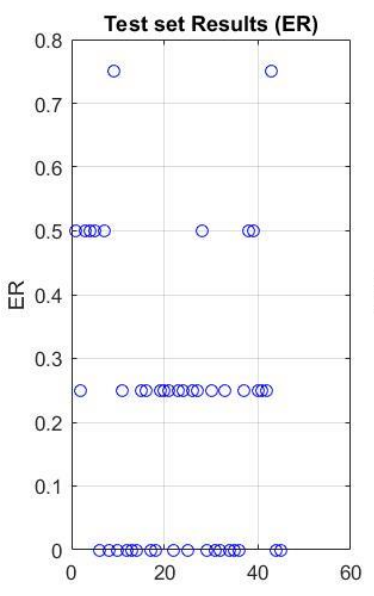

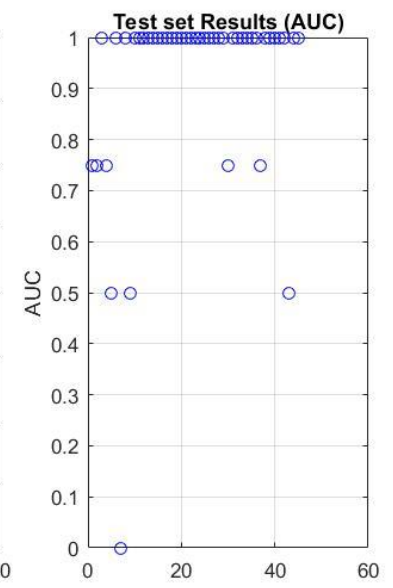

Negative mode
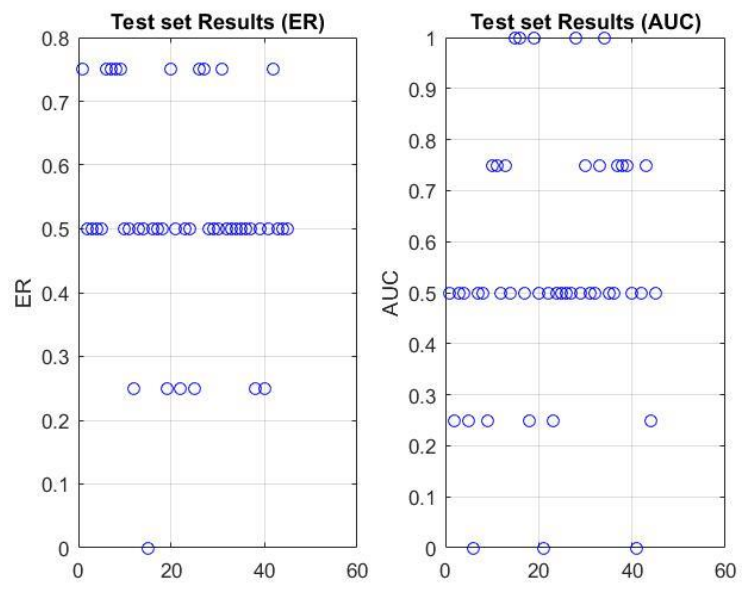


\section{Figure S3}

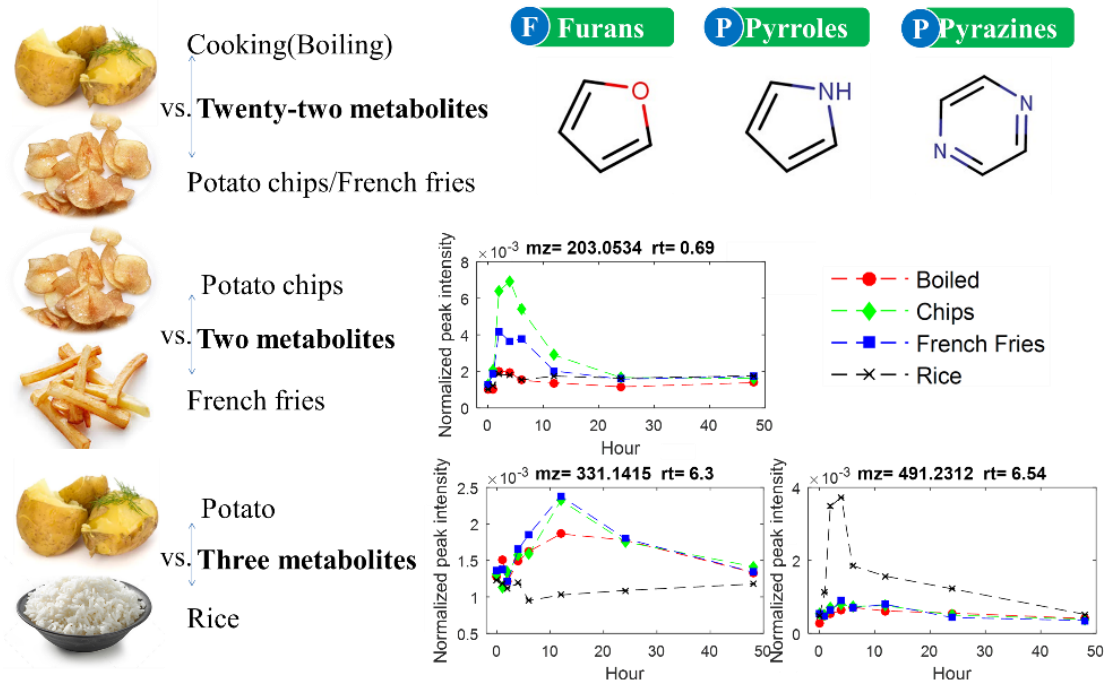

Figure S3. Overview of selected metabolites of intake for the respective food groups, including boiled potato (red line), potato chips (green line), French fries (blue line) and boiled rice (black line). 


\section{Figure S4}

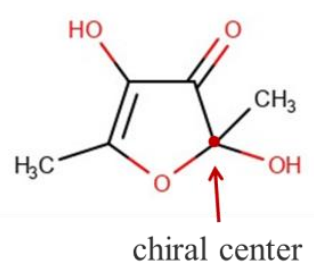

Figure S4. 2,4-dihydroxy-2,5-dimethyl-3(2H)-furanone with chiral center 


\section{Figure S5}

A $\mathrm{M} 1$

MS/MS 129.0547 at $28 \mathrm{eV}(\mathrm{ESI}+)$ in VION

(parent ion with $305.0888, \mathrm{C}_{12} \mathrm{H}_{16} \mathrm{O}_{9}$ )
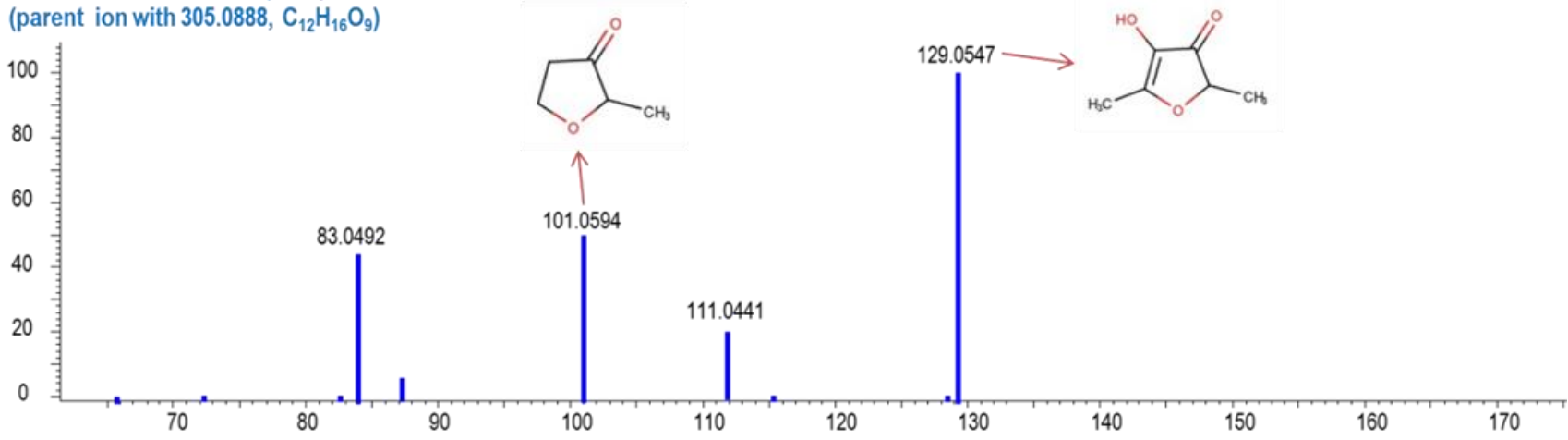

B M2-5

MS/MS 145.0497 at 14eV (ESI+) in VION
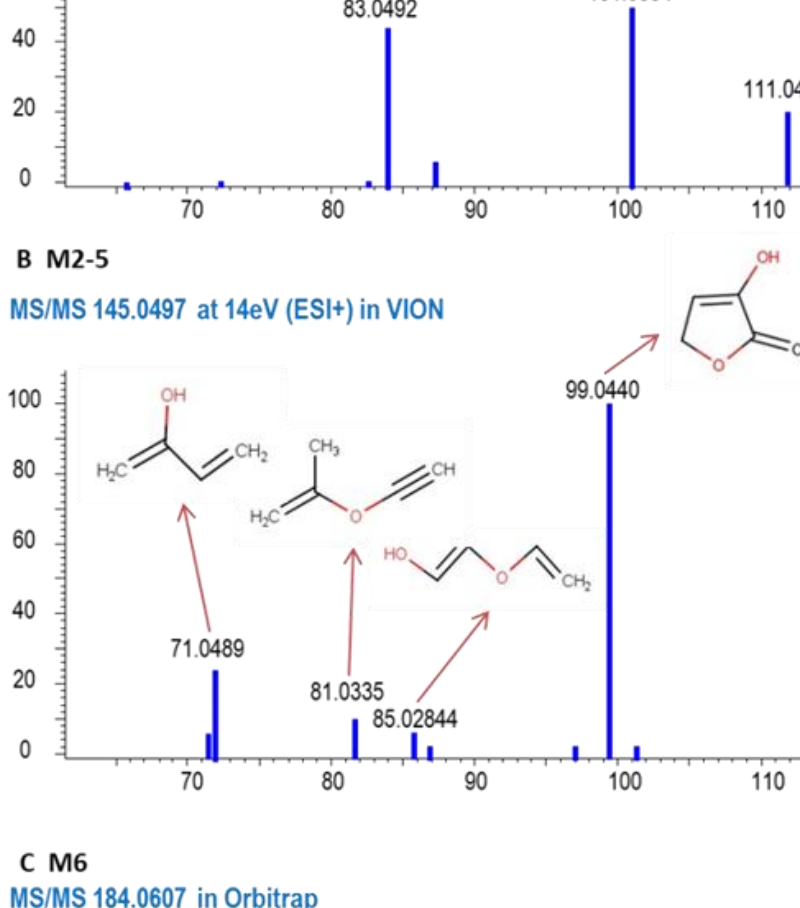

MS/MS 184.0607 in Orbitrap
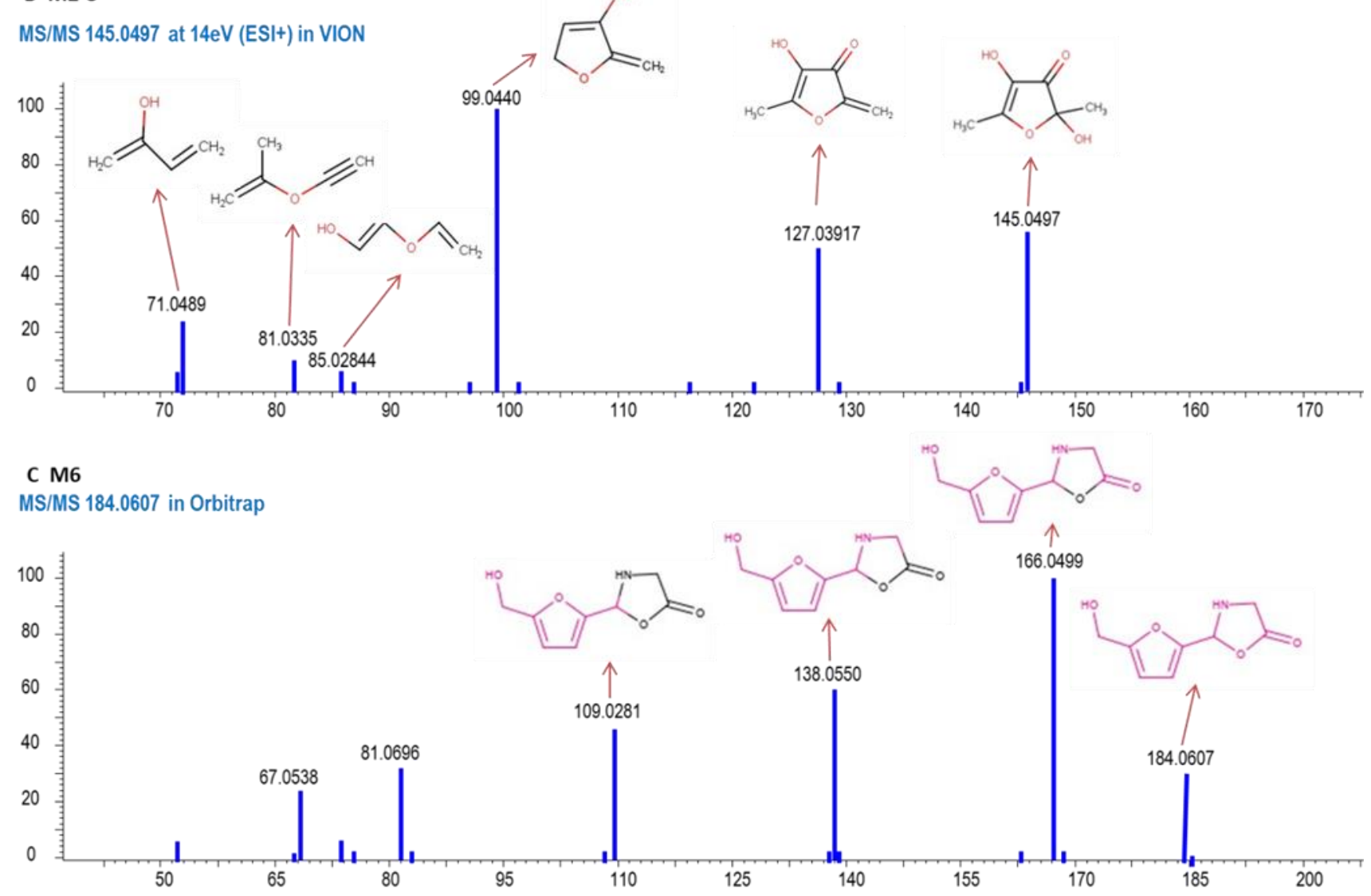

\section{M6}

MS/MS 184.0607 in Orbitrap
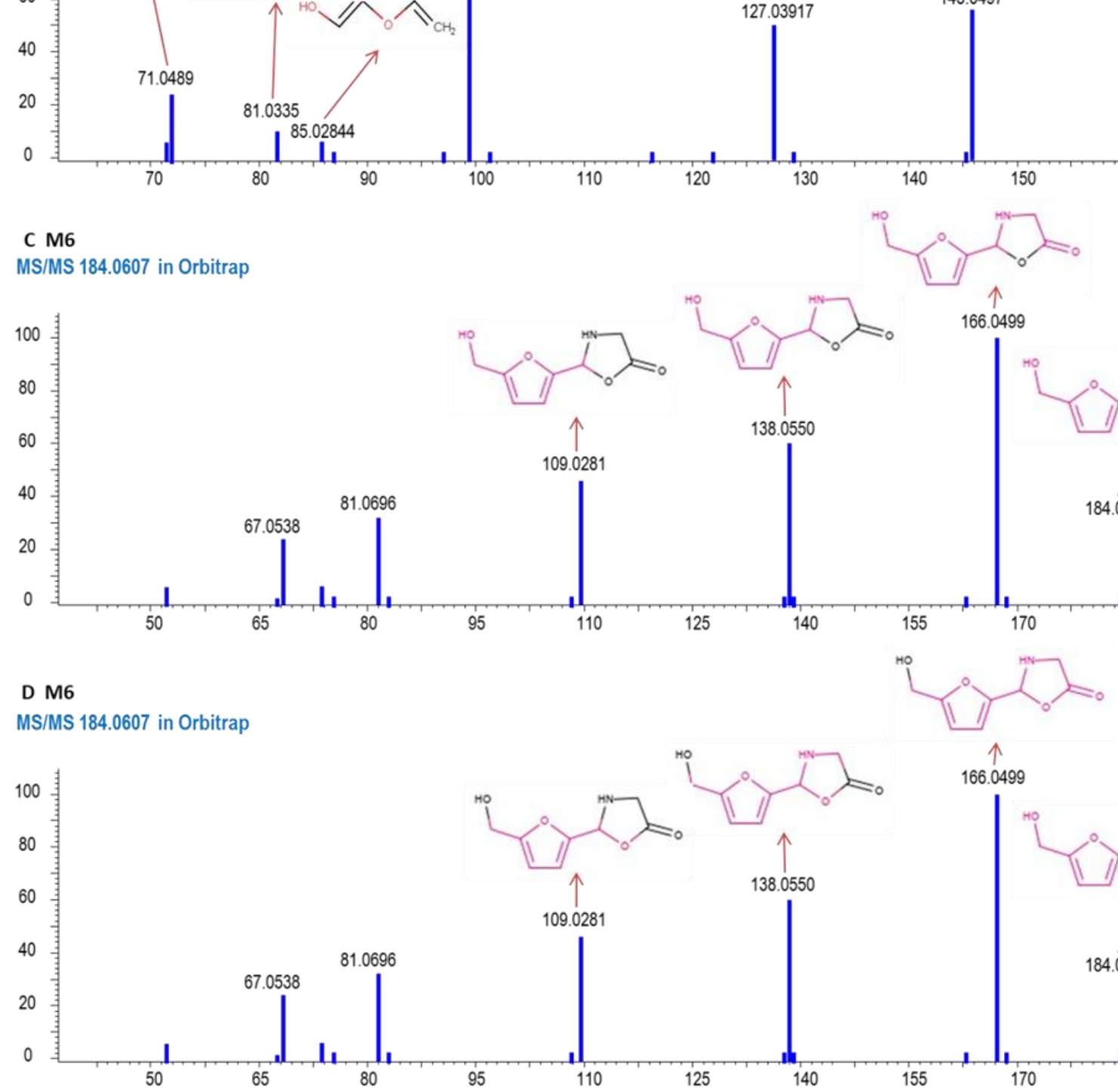


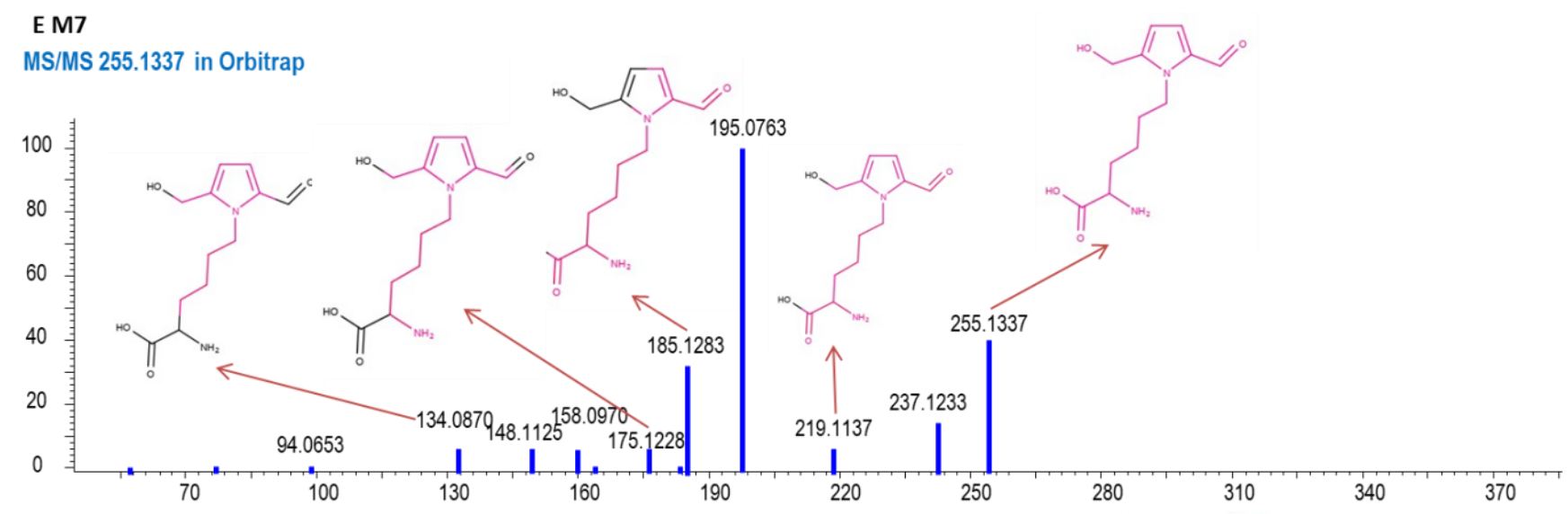

\section{F M8}

MS/MS 279.1479 at $14 \mathrm{eV}$ in VION
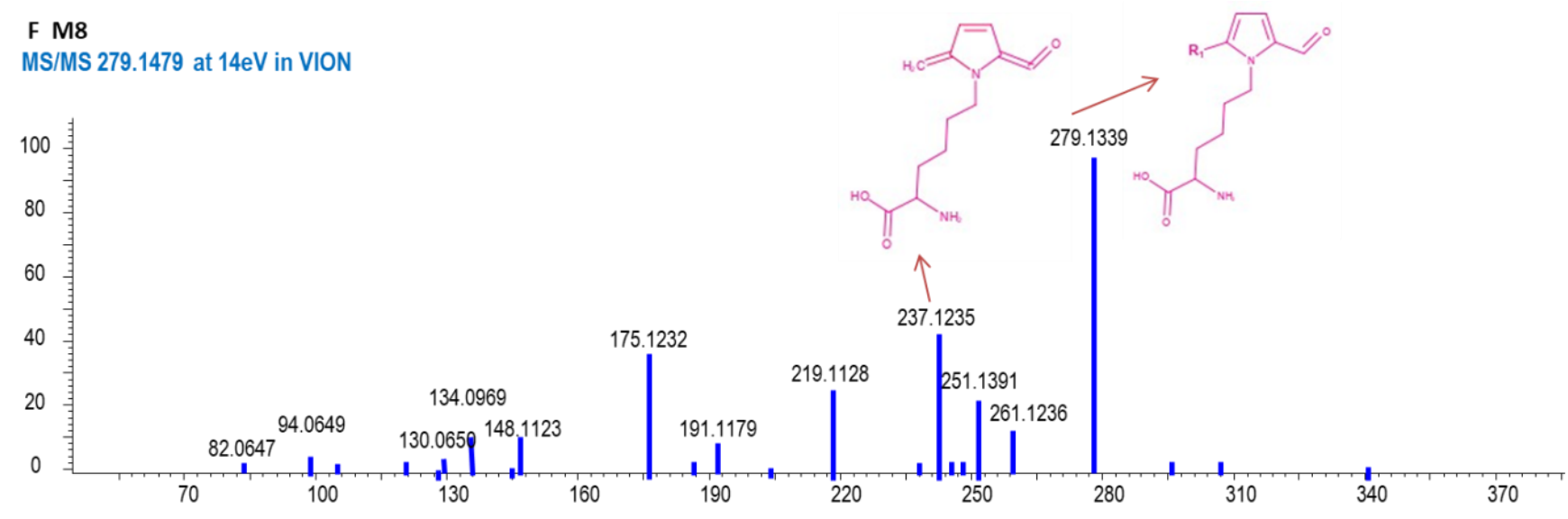

G M9
MS/MS 283.1348 in Orbitrap
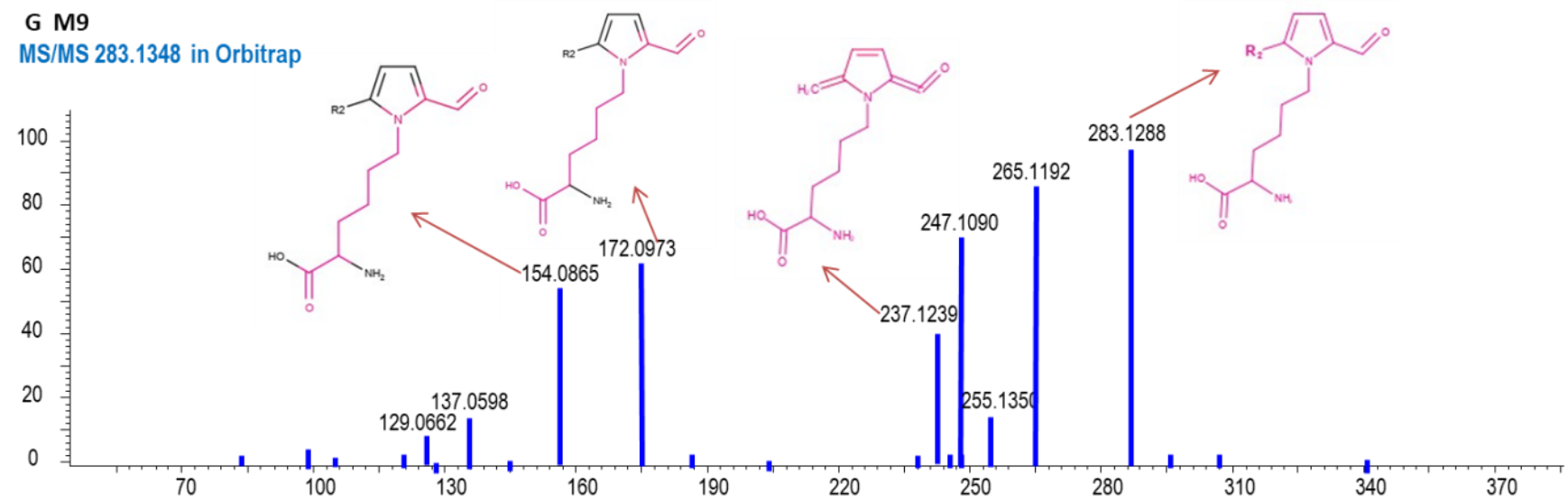

\section{H M10}

MS/MS 210.0759 at $28 \mathrm{eV}$ in VION
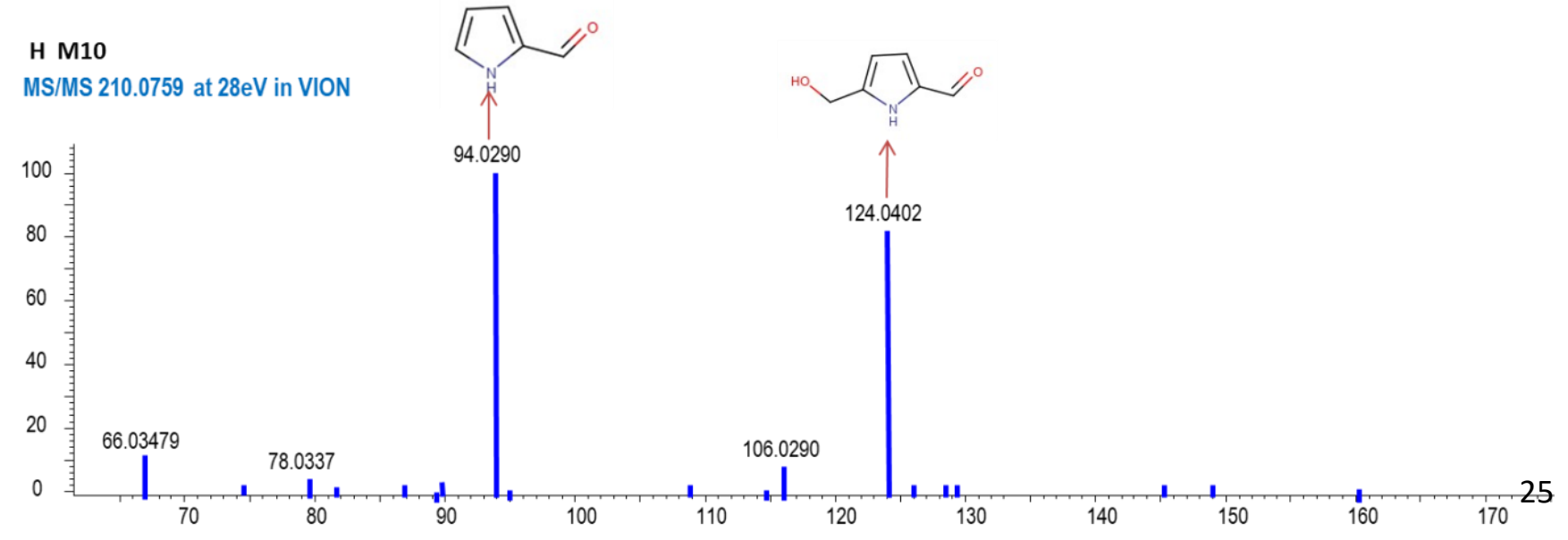

250 


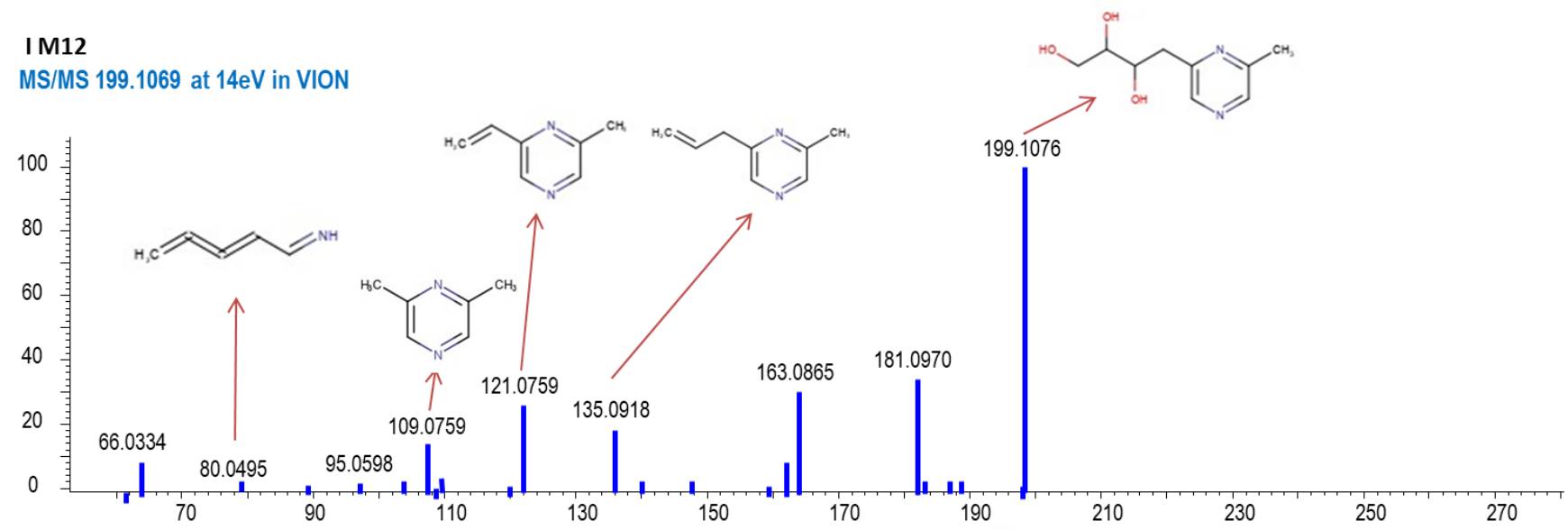

\section{J M13}

MS/MS 185.0927 at $14 \mathrm{eV}$ in VION
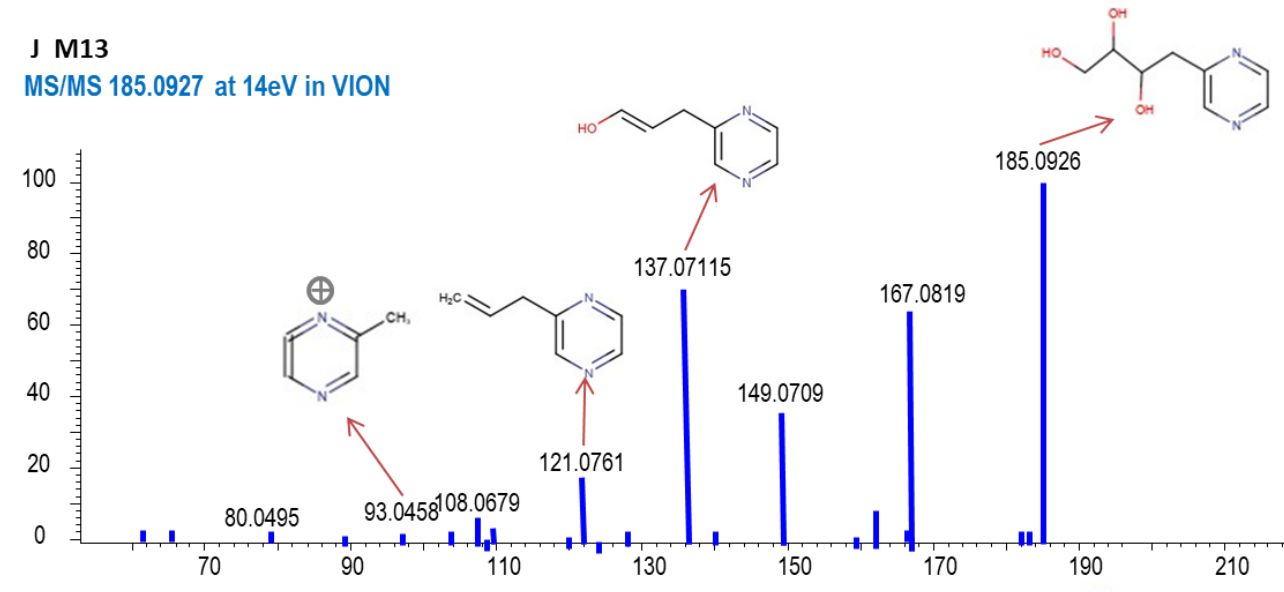

K M14

MS/MS 183.0753 at $14 \mathrm{eV}$ in VION
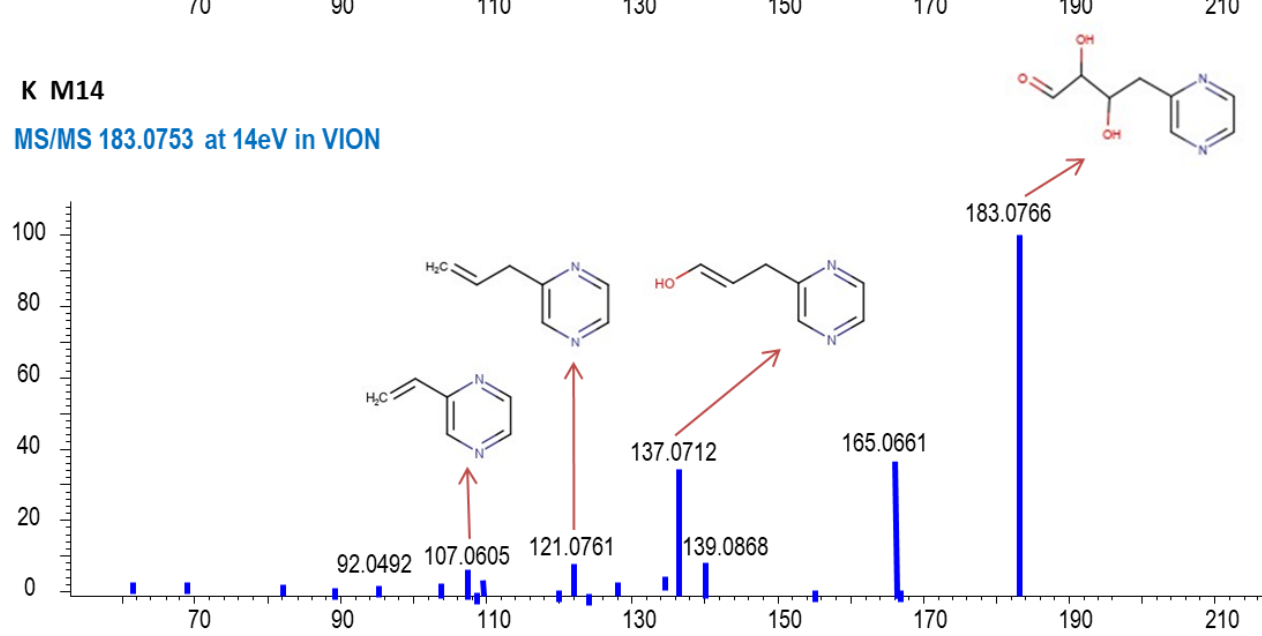

190

230

Figure S5. LC-MS/MS spectra of tentatively identified compounds obtained by LC-Qtof-MS and LTQ-Orbitrap. The proposed structures of product ions are entered in the mass spectra to indicate likely fragment identities. The structures of resulting product ions are highlighted in red color in C, D, E, F and G while neutral losses appear in black. The proposed structures may have alternatives, e.g. the fragmentation patterns of M6 in C and D show two ways to generate different structures with the same $\mathrm{m} / \mathrm{z}$. 


\section{Figure S6}

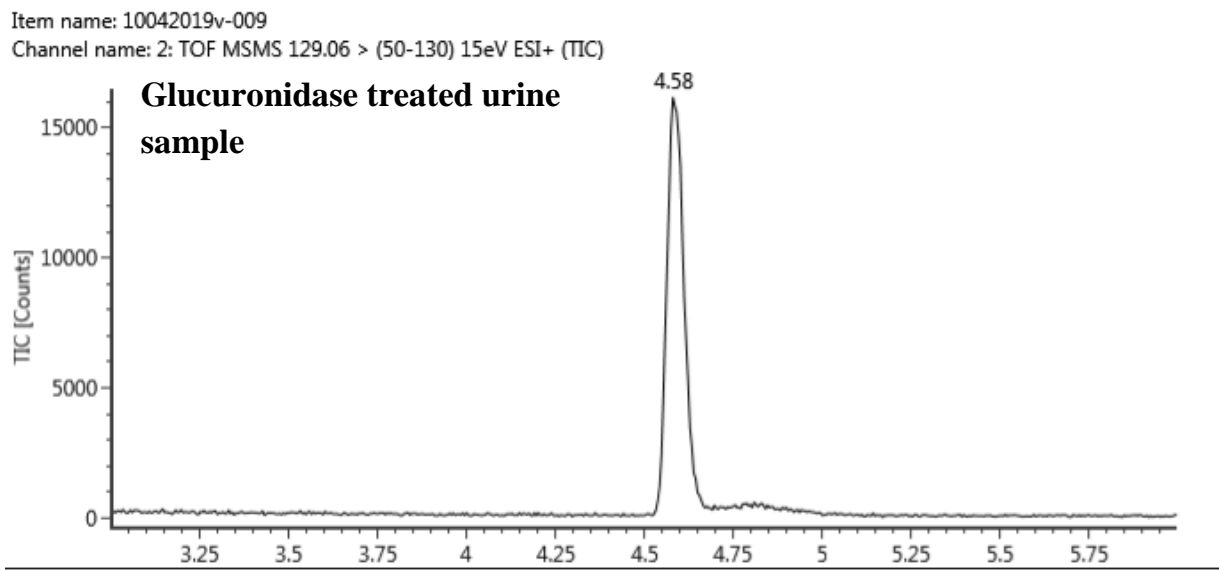

Item name: $10042019 \mathrm{v}-010$

Channel name: 2: +129.0550 (28.7 PPM) : TOF MSMS $129.06>(50-130) 15 \mathrm{eV}$ ESI+

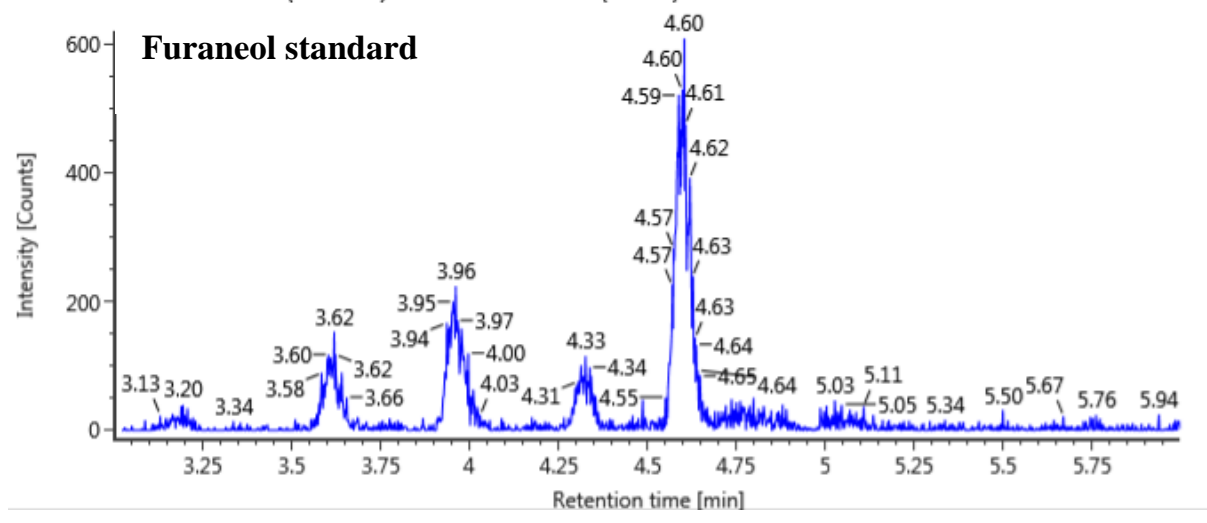

Item name: 10042019v-009 Channel name: 2: Average Time $4.5877 \mathrm{~min}:$ Set Mass $(\mathrm{m} / \mathrm{z})=129.0600:$ TOF MSMS $129.06>(50-130) 15 \mathrm{eV}$ ESI $:$ : Combined Item description: furaneol

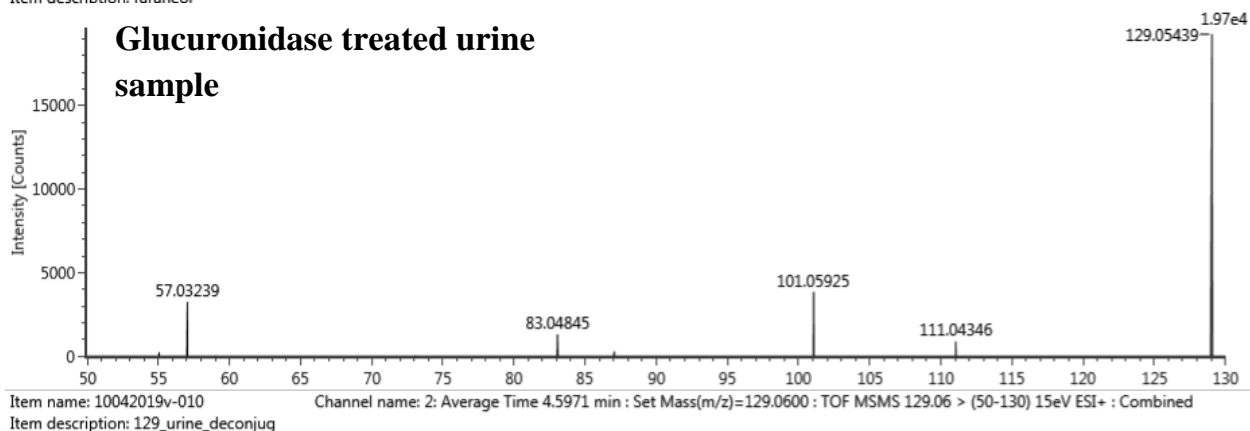
Item description: 129_urine_deconiug

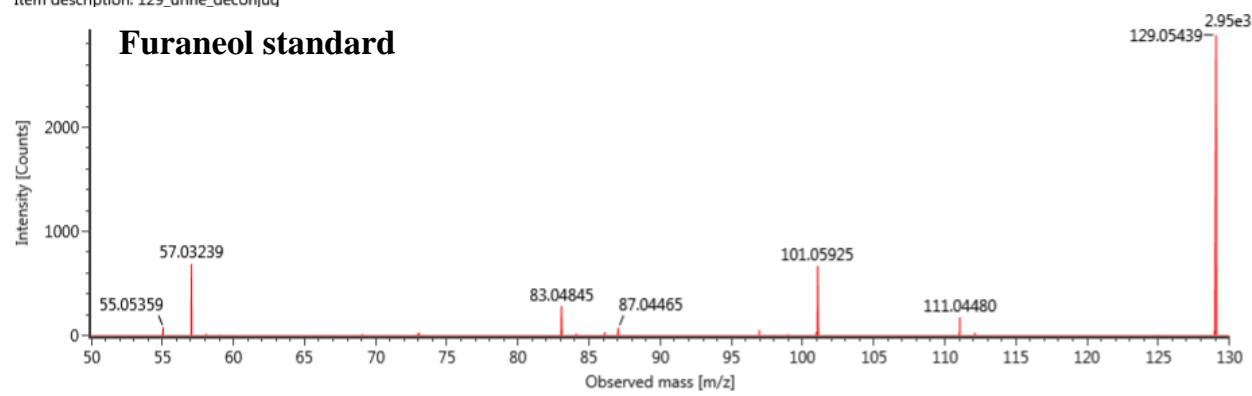


Figure S6 Extracted ion chromatogram and MS/MS spectrum at 14ev (ESI+) in Qtof of furaneol standard and deconjugated urine sample $2 \mathrm{~h}$ after potato chips intake. 


\section{Figure S7}

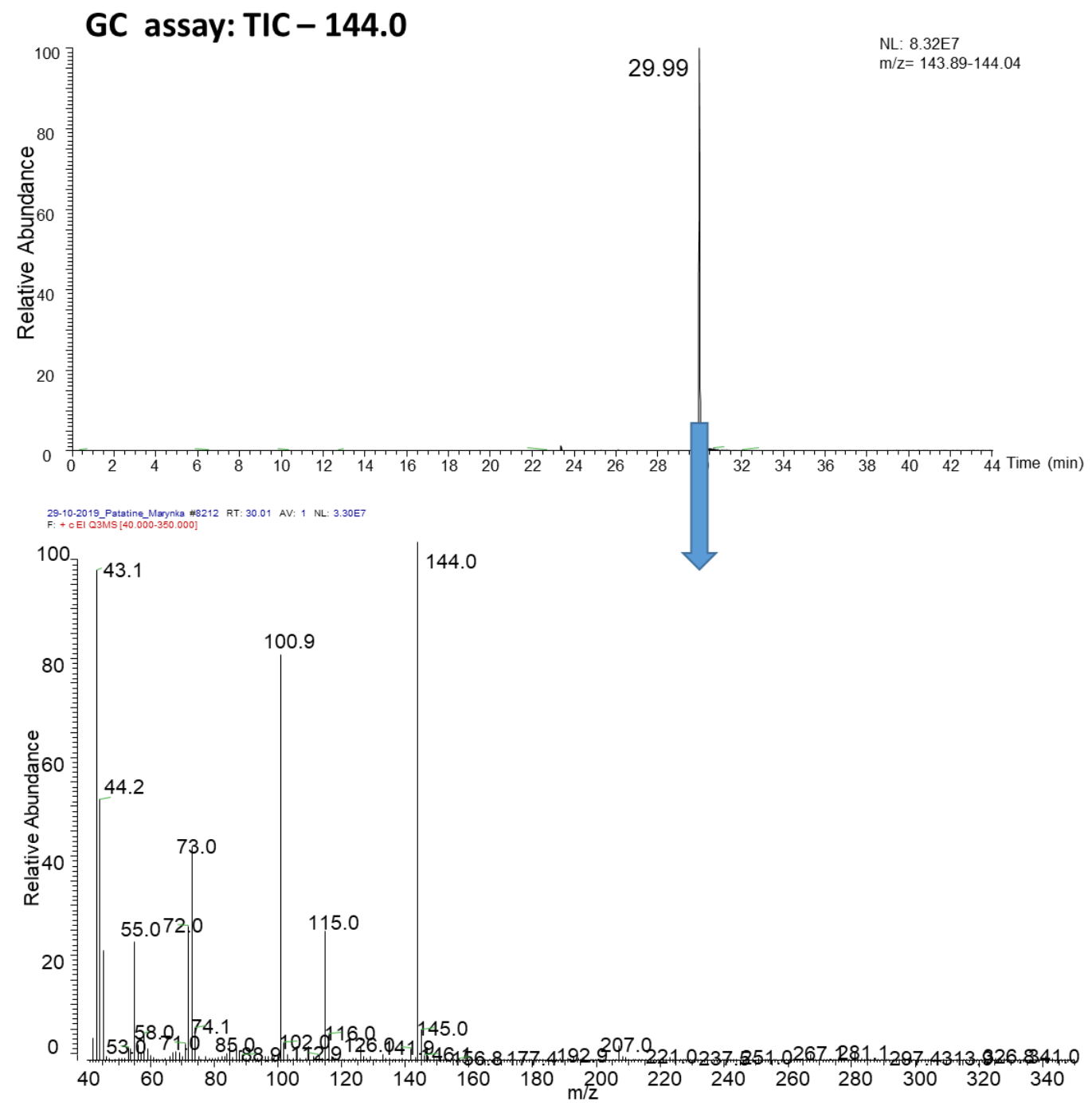




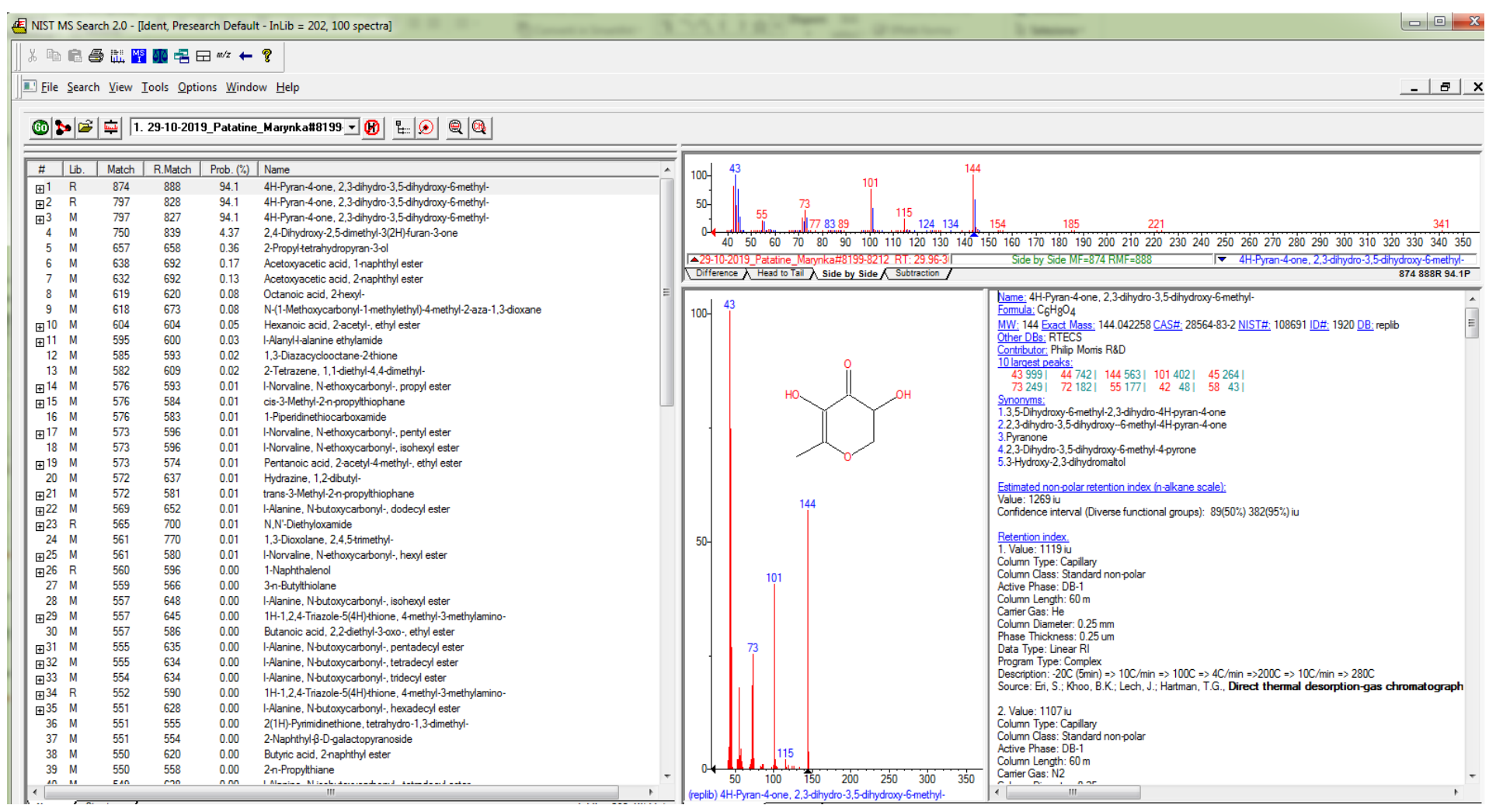

Figure S7. Chromatogram and mass spectra recorded using the GC-MS after injection of potato chips extract. The compound 144.0 RT 29.99 min was identified as 2, 3-dihydro-3, 5-dihydroxy-6-metryl-4H-pyran-4-one compared with NIST library. 


\section{Figure S8}

\section{Pyrraline standard}

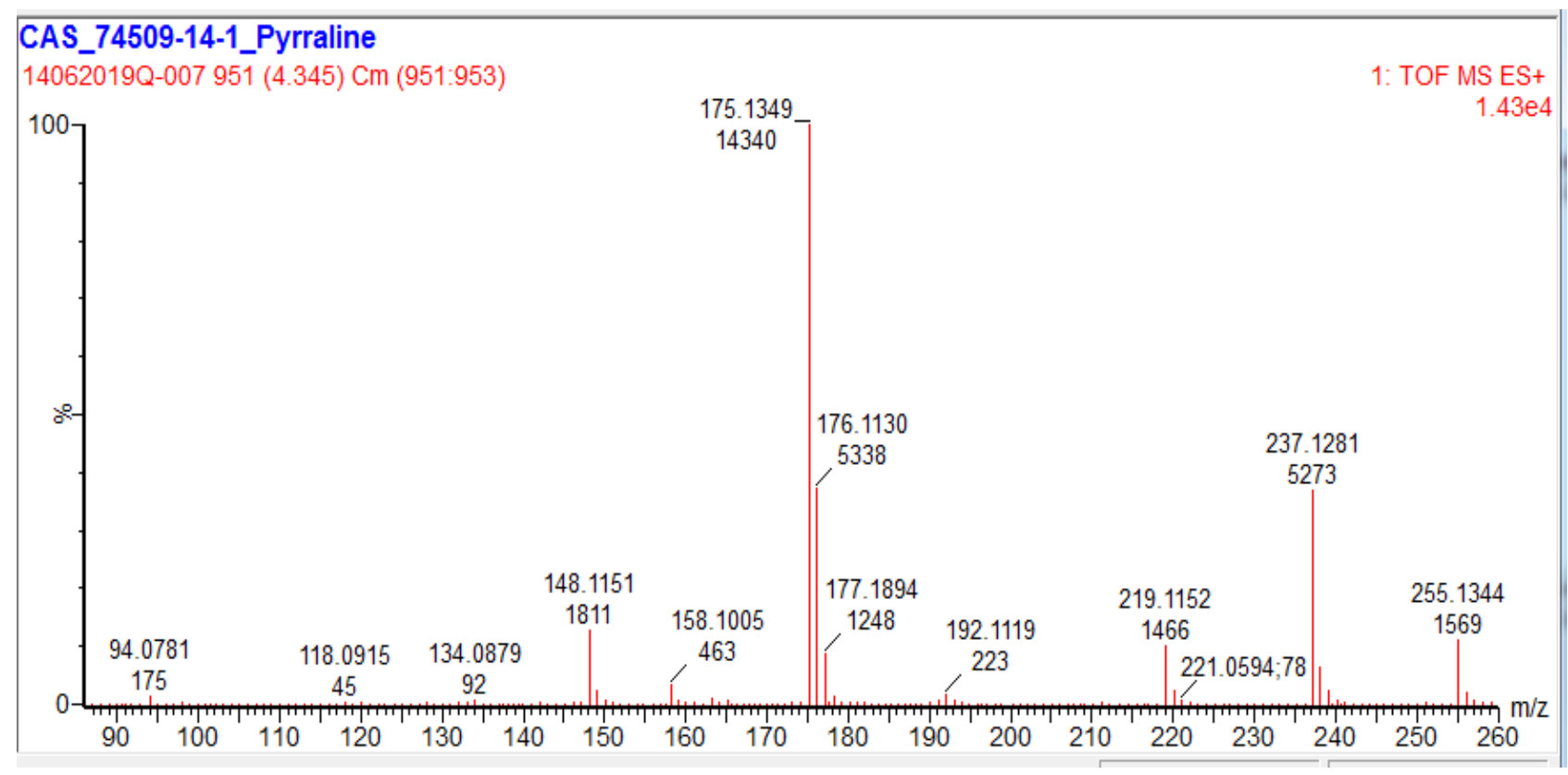

\section{Urine sample}

Item name: 20191127-V021

Item description: 29062017-158(6206-1-2)

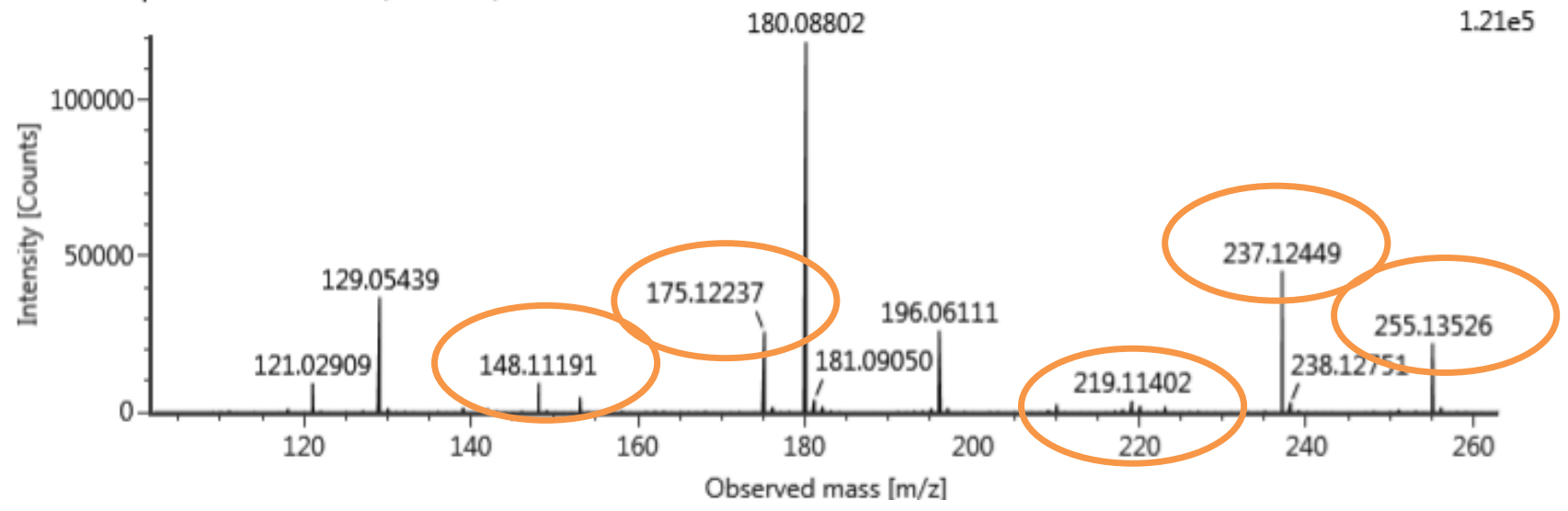

Figure S8. Extracted ion chromatogram and mass spectra recorded using the UPLC-Qtof-MS after injection of pyrraline standard as well as a urine sample collected 1-2 $\mathrm{h}$ after chips intake. The compound of $\mathrm{m} / \mathrm{z}[\mathrm{M}+\mathrm{H}]+$ 255.13 RT 4.27 was identified as pyrraline as level II based on fragmentation pattern by UPLC-Qtof-MS (mass detector is VION). 


\section{Supplemental references}

(1) Cuparencu, C.; Rinnan, Å.; Dragsted, L. O. Combined Markers to Assess Meat Intake-Human Metabolomic Studies of Discovery and Validation. Mol. Nutr. Food Res. 2019, 1900106. https://doi.org/10.1002/mnfr.201900106.

(2) Wakamatsu, J.; Stark, T. D.; Hofmann, T. Taste-Active Maillard Reaction Products in Roasted Garlic (Allium Sativum). J. Agric. Food Chem. 2016, 64 (29), 5845-5854. https://doi.org/10.1021/acs.jafc.6b02396.

(3) Roager, H. M.; Hansen, L. B. S.; Bahl, M. I.; Frandsen, H. L.; Carvalho, V.; Gøbel, R. J.; Dalgaard, M. D.; Plichta, D. R.; Sparholt, M. H.; Vestergaard, H.; et al. Colonic Transit Time Is Related to Bacterial Metabolism and Mucosal Turnover in the Gut. Nat. Microbiol. 2016, 1 (9), 1-9. https://doi.org/10.1038/nmicrobiol.2016.93. 